\title{
Transmit Power Policy and Ergodic Multicast Rate Analysis of Cognitive Radio Networks in Generalized Fading
}

\author{
Athira Subhash*, Muralikrishnan Srinivasan*, Sheetal Kalyani, Lajos Hanzo
}

\begin{abstract}
This paper determines the optimum secondary user (SU) power allocation and ergodic multicast rate of point-tomultipoint communication in a cognitive radio network (CRN) in the presence of various quality of service $(\mathrm{QOS})$ constraints for the primary users (PUs). Using tools from extreme value theory (EVT), it is first proved that the limiting distribution of the minimum of independent and identically distributed (i.i.d.) signal-to-interference ratio (SIR) random variables (RVs) is a Weibull distribution, when the user signal and the interferer signals undergo independent and non-identically distributed (i.n.i.d.) $\kappa-\mu$ shadowed fading. Also, the rate of convergence of the actual minimum distribution to the Weibull distribution is derived. This limiting distribution is then used for determining the optimum transmit power of a secondary network in an underlay CRN subject to three different QoS constraints at the primary network in a generalized fading scenario. Furthermore, the optimum transmit power and the asymptotic ergodic multicast rate of SUs is analyzed for varying channel fading parameters.
\end{abstract}

Index Terms-extreme value theory, $\kappa-\mu$ shadowed fading, cognitive radio, outage probability, queuing delay, secrecy outage

\section{INTRODUCTION}

With the advances in wireless technology, the presence of wireless devices has become ubiquitous. Furthermore, with the advent of the Internet of Things (IoT), the number of connected devices accessing the spectrum is set to increase in the upcoming times. With this increase in devices and hence increasing traffic, it will be very hard to find free spectrum. Cognitive radio (CR) is one of the promising techniques mitigating spectrum scarcity in wireless communication systems [1]-[5]. In cognitive radio networks (CRNs), there are three popular modes of spectrum sharing between primary users (PUs) and secondary users (SUs) - underlay, overlay and interweave [6]-[9]. As a further development, the authors of [10], [11] have studied the security aspects of a CR system in the presence of eavesdroppers.

Throughout this paper, we consider the underlay mode, where the secondary network accesses the same spectrum as

Athira Subhash, Muralikrishnan Srinivasan, and Sheetal Kalyani are with the Dept. of Electrical Engineering, Indian Institute of Technology, Madras, India. (Emails:\{ee14d206@ee,ee16d027@smail,skalyani@ee\}.iitm.ac.in).

Lajos Hanzo is with the School of Electronics and Computer Science, University of Southampton.(Email: hanzo@soton.ac.uk).

L. Hanzo would like to acknowledge the financial support of the Engineering and Physical Sciences Research Council projects EP/Noo4558/1, EP/PO34284/1, COALESCE, of the Royal Society's Global Challenges Research Fund Grant as well as of the European Research Council's Advanced Fellow Grant QuantCom

*Athira Subhash and Muralikrishnan Srinivasan are co-first authors. the primary network. An important problem in CRNs is the choice of power policy at the SU-Tx (transmitter), so that the interference at the PU-Rx (receiver) remains below an affordable threshold. Several authors [12]-[18] have studied the performance of underlay CRNs under various interference constraints. Similarly, recent contributions [19], [20] have also considered the performance of an interference-limited underlay CRN relying on continuous power adaptation at the SU. In [20], the SU-Tx is assumed to transmit information to the specific SU-Rx (receiver), having the $k$ th highest signal-tointerference ratio (SIR). The authors of [5] study the optimal power allocation, the effective number of SU-Tx antennas, the trade-off between transmit-and-harvest secondary antennas, and the average channel capacity of an energy harvesting (EH)-aided secondary system in a massive multiple input multiple output (MIMO) CRN.

Most of the above contributions concentrate on maintaining a minimum outage or rate at the PUs. However, in delaysensitive applications satisfying the delay constraint at the PU is of paramount importance. The delay constraints of PUs are not captured explicitly by the outage constraints, since the relationship between the delay imposed by the PU's queue and its outage probability is not explicit. Moreover, for delaysensitive devices, a simple outage or rate constraint cannot ensure that the data is not accumulated or overflowing in the output buffer. Therefore, it becomes necessary to incorporate some delay-based quality of service (QoS) constraint in our resource allocation problems [21], [22]. Hence we also solve the SU-Tx power allocation problem under a delay constraint at the PU-Rxs.

Another typical concern in wireless networks is the physical-layer security and protection of important nodes from eavesdroppers. The security of cognitive radio networks (CRNs) from attacks by eavesdroppers at the primary or secondary nodes is of high interest and has been studied in [23]-[25]. If the statistical information regarding the links between the eavesdropper and any critical node is available, then optimal power allocation can be performed for ensuring that the security of the link is not compromised. This is mathematically captured by the secrecy outage probability of the critical node. In this treatise we also discuss results that can be used for maintaining the required secrecy constraints at the PU-Rxs).

The authors of [19], [20] consider the analysis of transmit power policy at the SU and the ergodic capacity of the SU in Rayleigh fading channels. Our focus in this treatise is on 
extending these results to general fading scenarios. At the time of writing, generalized multipath fading models such as the $\kappa-\mu$ and the $\eta-\mu$ fading distributions are generating significant research interests [26]. They model the small-scale variations in the fading channel in line of sight (LOS) and non-line of sight (NLOS) conditions respectively [27]. To investigate the effects of shadowing on the dominant LOS component, the authors of [27] and [28] have developed a generalization of the shadowed Rician fading called the $\kappa-\mu$ shadowed fading model. The $\kappa-\mu$ shadowed fading has been shown to unify the $\kappa-\mu$ and $\eta-\mu$ fading models [29] and to have a wide variety of applications ranging from landmobile satellite systems to device-to-device communication [28]. Performance metrics conceived for generalized fading have been studied extensively in [30]-[45]. Recently, the authors of [46] studied the exact outage and rate expressions in an interference-limited scenario.

Calculating the outage/secrecy/queuing delay constraints over several PU-Rx requires the knowledge of the cumulative distribution function (CDF) of the minimum SIR among the mulicast users [19], [20]. Furthermore, the ergodic rate of the multicast scheme in the secondary network is determined directly by the SIR of the weakest user. Now, if we have to evaluate the exact expression for the complementary cumulative distribution function (CCDF) of minimum of say $L$ random variables (RVs), we have to evaluate the product of the CCDF of $L$ such RVs. Note that the expressions for the probability distribution function (PDF)/CDF of signal to interference ratio (SIR)/signal to noise ratio (SNR)/signal to interference plus noise ratio (SINR) in generalized fading scenarios have complicated expressions [32], [37]-[41], [43], [44], [46]. Hence, evaluating the exact expression of the statistics of extremes is difficult, particularly for moderate to large values of $L$ [51]. Thus, it is imperative that a simple limiting distribution is found for the minimum of SIR RVs in generalized fading scenarios.

Tools from Extreme Value Theory (EVT) are commonly used for characterizing the distributions of extremes and the peaks over thresholds. A brief list of some of the key literature using EVT for studying the limiting distribution of SIR in wireless systems is available in [51]. Against this backdrop, in this contribution we use EVT to determine the power adaptation at the SU underlay in an CRN, subject to specific QoS constraints for the PUs. We also use EVT for determining the ergodic multicast rate of the SUs. In Table I, we provide a bold summary and comparison of the seminal literature relying on system models similar to our scenario. Our main contributions in this paper are as follows:

- Assuming that the user signal and the interferer signal undergo independent and non-identically distributed (i.n.i.d.) $\kappa-\mu$ shadowed fading, we prove that the limiting distribution of the minimum of $L$ such independent and identically distributed (i.i.d.) SIR RVs is a Weibull distribution.

- We also derive the rate of convergence of the actual distribution of the minimum SIR to the derived asymptotic distribution.

- Using the limiting distribution derived, we determine a closed form expression for the optimum power to be used at the SU-Txs while the PU-Rx are subjected to three different QoS constraints.

- Furthermore, we derive expressions for the ergodic multicast rate of point-to-multipoint communications in the secondary network.

Note that the results presented above can be readily used for the analysis of many other common channel fading models, which are special cases of the $\kappa-\mu$ shadowed fading.

\section{SySTEM MODEL}

We consider a CR scenario where the PU network consists of a PUTx serving $M$ multicast PU-Rxs and a SU network that consists of a SU-Tx serving $L$ multicast SURxs. Here, all the devices have a single antenna for transmission/reception. Furthermore, here we assume that the SUTx sends common multicast information to all the SU-Rxs in the underlay mode. Since an underlay mode is considered, the SU-Tx has to rely on continuous power adaptation strategy for satisfying the QoS constraints at the PU-Rxs. The channel power gains of the links PUTx $\rightarrow$ PURx $_{m}$, for $m=1,2,, M$ and SUTx $\rightarrow$ $\mathrm{SURx}_{l}$, for $l=1,2, L$ are denoted by $h_{m}$, for $m=1,2, M$ and $g_{l}$, for $l=1,2, L$, respectively. Similarly, $\alpha_{m}$ and $\beta_{l}$ are the channel power gains of the interference links SUTx $\rightarrow$ PURx $_{m}$ and PUTx $\rightarrow$ SURx $_{l}$, respectively. All the channels are considered to undergo $\kappa-\mu$ shadowed fading. Furthermore, we consider an interference-limited system, where the noise power at each of the SU-Rx (or PU-Rx) is negligible compared to the interference power received from the PU-Tx (or SU-Tx). The authors of [19], [20] consider a similar system model except for the fact that they assume Rayleigh faded channels. Furthermore, the authors of [20] consider only one PU-Rx. The instantaneous SIRs at the $m$ th PURx and $l$ th SURx are

$$
\gamma_{m, p}=\frac{P_{p} h_{m}}{P_{s} \alpha_{m}}, \quad m=1, \ldots, M
$$

and

$$
\gamma_{l, s}=\frac{P_{s} g_{l}}{P_{p} \beta_{l}}, \quad l=1, \ldots, L,
$$

respectively. Here, $P_{p}$ is the PUTx transmit power, $P_{s}$ is the instantaneous SUTx transmit power and $\left\{h_{m}, \alpha_{m}, g_{l}, \beta_{l} ; m=\right.$ $1,2,, M, l=1,2,, L\}$ are $\kappa-\mu$ shadowed random variables (RVs). A $\kappa-\mu$ shadowed RV $X$ with parameters $(\kappa, \mu, m, \bar{x})$ has the following pdf [27]:

$$
f_{X}(x)=\frac{x^{\mu-1}}{\theta^{\mu-m} \lambda^{m} \Gamma[\mu]} e^{-\frac{x}{\theta}}{ }_{1} F_{1}\left(m, \mu, \frac{x}{\theta}-\frac{x}{\lambda}\right), x \geq 0
$$

where ${ }_{1} F_{1}($.$) is the confluent hypergeometric function, \Gamma[$. is the gamma function, $\theta=\frac{\bar{x}}{\mu(1+\kappa)}, \lambda=\frac{(\mu \kappa+m) \bar{x}}{\mu(1+\kappa) m}$ and $\bar{x}=\mathbb{E}[X]$. Here, $\mathbb{E}[$.$] represents the expectation of a RV.$ Throughout this paper we assume that the CSIs of the links are not estimated frequently, but the statistics of the signal and interference links are known at the transmitters. 


\begin{tabular}{|c|c|c|c|c|c|c|c|c|}
\hline & Our model & [20]-2019 & [47]-2018 & [19]-2016 & [48]-2015 & [15]-2011 & [49]-2016 & {$[50]-2015$} \\
\hline No of PU-Rx & Multiple & Single & Single & Multiple & Multiple & Single & Single & Single \\
\hline No of SU-Rx & Multiple & Multiple & Single & Multiple & Single & Single & Single & Single \\
\hline $\mathbf{I} / \mathbf{S}^{1}$ & $\mathrm{~S}$ & $\begin{array}{l}\text { Su-Tx to } \\
\text { Pu-Rx:I, } \\
\text { Rest of the } \\
\text { links:S }\end{array}$ & both & $\mathrm{S}$ & I & I & I & $\mathrm{S}$ \\
\hline $\begin{array}{l}\text { Channel } \\
\text { Fading }\end{array}$ & $\begin{array}{l}\kappa-\mu \text { shad- } \\
\text { owed }\end{array}$ & Nakagami & Rayleigh & Rayleigh & Nakagami & - & - & Rayleigh \\
\hline I-Pu-Tx ${ }^{2}$ & $\checkmark$ & $\checkmark$ & $x$ & $\checkmark$ & $x$ & $\checkmark$ & $\checkmark$ & $\checkmark$ \\
\hline $\begin{array}{l}\text { QoS } \\
\text { constraints }\end{array}$ & $\begin{array}{l}\text { Probability } \\
\text { of outage } \\
\text { at Pu-Rx, } \\
\text { secrecy } \\
\text { outage, } \\
\text { Delay QoS }\end{array}$ & $\begin{array}{l}\text { Interference } \\
\text { to } \mathrm{Pu}-\mathrm{Rx}\end{array}$ & $x$ & $\begin{array}{l}\text { Interference } \\
\text { to } \mathrm{Pu}-\mathrm{Rx}\end{array}$ & - & $\begin{array}{l}\text { Interference } \\
\text { to } \mathrm{Pu}-\mathrm{Rx}\end{array}$ & $\begin{array}{l}\text { Secrecy } \\
\text { outage }\end{array}$ & Delay QoS \\
\hline $\begin{array}{l}\text { Usage of } \\
\text { EVT }\end{array}$ & $\checkmark$ & $\checkmark$ & $x$ & $x$ & $x$ & $x$ & $x$ & $x$ \\
\hline $\begin{array}{l}\text { Expression } \\
\text { for } \\
\text { secondary } \\
\text { capacity }\end{array}$ & $\checkmark$ & $x$ & $x$ & $\checkmark$ & $x$ & $x$ & $x$ & $x$ \\
\hline
\end{tabular}

TABLE I: Comparison with existing literature.

\section{SECONDARY USER POWER CONTROL POLICY}

In the underlay mode, the SU-Tx transmits over the same frequency as the PU-Tx, even when the PU-Tx is active. Simultaneous transmission occurs as long as the QoS degradation at the PU-Rx due to the interference imposed by the SUTx is tolerable. This QoS degradation in the primary network is quantified by means of different constraints at the PURxs. In this contribution, we consider the following three QoS constraints :

- Outage constraint at the PU-Rxs,

- Queuing delay constraint at the PU-Rxs,

- Secrecy outage constraint at the PU-Rx.

In the subsequent subsections we discuss each of these constraints separately.

\section{A. Outage constraint at the PU-Rx}

Here, the SU-Tx must transmit at a power that keeps the outage at each of the PU-Rx below a predetermined level. Thus, transmit power policy at the SU-Tx can be mathematically formulated as follows [19], [20],

$$
\begin{aligned}
& \max P_{s}, \\
\text { s.t. } & \mathbb{P}\left\{\gamma_{m, p}\left(P_{s}\right) \leq \gamma_{0}\right\} \leq p_{0}, \quad \forall m=1, \ldots, M \\
& P_{s} \leq P_{s, \max },
\end{aligned}
$$

where $p_{0}$ is the maximum tolerable outage at each of the PU$\mathrm{Rx}$ and $\gamma_{0}$ is the minimum desired SIR at the PU-Rx for a fixed PU transmit power $P_{p}$. The outage constraint in (4b) is equivalent to the condition where $\mathrm{PU}-\mathrm{Rx}_{m}$ with the lowest SIR satisfy the outage constraint. Hence, the power policy of SU-Tx can be alternatively formulated as

$$
\begin{array}{ll} 
& \max P_{s} \\
\text { s.t. } & \mathbb{P}\left\{\min _{1 \leq m \leq M} \gamma_{m, p}\left(P_{s}\right) \leq \gamma_{0}\right\} \leq p_{0} \\
& P_{s} \leq P_{s, \max } .
\end{array}
$$

Substituting the fading coefficients from (1) into (5b), we obtain

$$
\begin{array}{ll} 
& \max P_{s} \\
\text { s.t. } & \mathbb{P}\left\{\min _{1 \leq m \leq M} \frac{h_{m}}{\alpha_{m}} \leq \gamma_{0} \frac{P_{s}}{P_{p}}\right\} \leq p_{0} . \\
& P_{s} \leq P_{s, \text { max }} .
\end{array}
$$

Here, $\left\{h_{m} ; m=1, \cdots, M\right\}$ and $\left\{\alpha_{m} ; m=1, \cdots, M\right\}$ are sets of i.i.d. $\kappa-\mu$ shadowed RVs with fading parameters $\left(\kappa_{p}, \mu_{p}, m_{p}, \bar{h}_{p}\right)$ and $\left(\kappa_{p, s}, \mu_{p, s}, m_{p, s}, \bar{\alpha}_{p, s}\right)$ respectively. Note that a more realistic model would rely on non-identical links between the transmitter and multiple receivers. However, analyzing this scenario is intractable due to the complex nature of the CCDF in generalized fading scenarios. The assumption of identical links holds true in scenarios where the users are in a stationary environment, such as ad-hoc networks in buildings or in case of slowly moving users [19]. Similar, simplified models are widely used for the performance analysis of CR systems [19], [20], [52]-[54]. The abovementioned contributions analyze the performance of different CR systems assuming identical links between the transmitters and receivers. Therefore, even the study of the statistics of the minimum SIR over i.i.d. links is relevant and will hopefully serve as a spring-board for more general analysis.

To determine the optimum value of $P_{s}$ that satisfies the outage constraint in $(5 \mathrm{~b})$, we have to determine the CDF of the minimum of SIR RVs in a $\kappa-\mu$ shadowed fading environment. Note that, we can evaluate this using the CDF of the minimum of ratio of two $\kappa-\mu$ shadowed RVs as given in (6b). The exact distribution of the minimum of any set of i.i.d. RVs $\gamma_{\min }=$ $\min \left\{\gamma_{1}, \gamma_{2}, \cdots, \gamma_{M}\right\}$, where $\gamma_{i} \sim F_{\gamma}(z) ; \forall i \in\{1, \cdots, M\}$ is given by

$$
F_{\gamma_{\min }}(z)=1-\left(1-F_{\gamma}(z)\right)^{M}
$$

Hence, to evaluate the CDF in (6b), we have to evaluate the $M^{t h}$ power of the CCDF of ratio of $\kappa-\mu$ shadowed RVs. The exact expression for the CDF of ratio of $\kappa-\mu$ shadowed RVs 
is given in terms of an infinite sum of the Lauricellas function of the fourth kind in [55, Eq. 3], [46]. The complex nature of the CDF $F_{\gamma}(z)$ makes the evaluation of the $M^{t h}$ power of the CDF difficult. Now, even if we find an approximation for the CDF of $\gamma$, any small error in the computation of $F_{\gamma}(z)$ will become amplified due to the exponent to which it is raised and hence it will make the corresponding distribution function less accurate. Note that even if we compute the exact distribution for large values of $M$, it will not be possible to derive any meaningful inference from them owing to the complex nature of those expressions.

On the other hand, if we have a simple limiting distribution for (7), which closely approximates the CDF values for moderate and large values of $M$, we can obtain a closed-form expression for the optimum $P_{s}$ that satisfies (6b). For small values of $M$ we can still use the exact CDF of the minimum. Therefore, using tools from EVT, we formulate the following theorem to determine the limiting distribution of (7), when $\gamma$ is the SIR in an $\kappa-\mu$ shadowed fading environment. We then use this theorem to evaluate the probability expression in (6b) and hence obtain a closed-form expression for the optimum $P_{s}$. A similar approach is used for determining the ergodic multicast rate of the secondary users in [20] for Rayleigh faded channels. To the best of our knowledge, no previous work has used EVT to simplify the outage constraints at the PU-Rx in a generalized fading scenario.

Theorem 1. Consider $K$ i.i.d. SIR RVs of the form

$$
\gamma_{k}=\frac{d_{k}}{\sum_{j=1}^{N} c_{j, k}}
$$

where $\left\{d_{k} ; 1 \leq k \leq K,\right\}$ are i.i.d. $\kappa-\mu$ shadowed $R V s$ with parameters $(\kappa, \mu, m, \bar{x})$ and $\left\{c_{j, k} ; 1 \leq j \leq N\right\}$ are i.n.i.d. $\kappa-\mu$ shadowed RVs, with parameters $\left(\kappa_{j}, \mu_{j}, m_{j}, \bar{x}_{j}\right)$ $\forall k \in\{1, \cdots, K\}$, for $j \in\{1, \cdots, N\}$. Here, $N$ is the number of interferers. The asymptotic distribution of $\gamma_{\text {min }}^{K}=\min \left\{\right.$ gamma $\left._{1}, \gamma_{2}, \ldots, \gamma_{K}\right\}$ is a Weibull distribution having the shape parameter $v=\mu$ and scale parameter $a_{K}=F_{\gamma}^{-1}\left(\frac{1}{K}\right)$, where $F_{\gamma}(z)$ is the common CDF of i.i.d. $R V s \gamma_{k}$. Let, $\gamma_{\min }=\lim _{K \rightarrow \infty} \gamma_{\min }^{K}$, then we have,

$$
F_{\gamma_{\text {min }}}(z)= \begin{cases}1-\exp \left(-\left(z / a_{K}\right)^{v}\right), & z \geq 0, \\ 0, & z<0 .\end{cases}
$$

Proof. Please refer to Appendix A for the proof.

To evaluate $a_{K}$, an approximation of the $\operatorname{CDF} F_{\gamma}(z)$ relying on the Lauricella function of the forth kind given by [46, Eqn 8] is used. Furthermore, [46] gives bounds on the truncation error and shows that the CDF is well approximated by the proposed expression. Finally, the MATLAB code for evaluating Lauricellas function of the fourth kind is available in [56].

Note that the above expression is simpler to evaluate than the actual CDF of the minimum as given in (7). Fig.1 shows the simulated and theoretical asymptotic CDF of minimum over $K=20$ SIR RVs for different system parameters. Here, cases 1, 2 and 3 correspond to the channel fading parameters as given in Table II. The results indicate that the asymptotic results are close to the true minimum distribution even for the cases where the minimum is evaluated over moderate-length sequences, such as $K=20$.

\begin{tabular}{|c|c|c|c|c|c|c|c|}
\hline Case \# & $\kappa$ & $\mu$ & $m$ & $N$ & $\left\{\kappa_{i}\right\}$ & $\left\{\mu_{i}\right\}$ & $\left\{m_{i}\right\}$ \\
\hline 1 & 2 & 3 & 1 & 3 & $\{2,2,2\}$ & $\{2,2,2\}$ & $\{1,1,1\}$ \\
\hline 2 & 2 & 3 & 1 & 2 & $\{2,2\}$ & $\{2,1\}$ & $\{1,1\}$ \\
\hline 3 & 2 & 2 & 1 & 1 & $\{2\}$ & $\{1\}$ & $\{1\}$ \\
\hline
\end{tabular}

TABLE II: Simulation parameters used for Fig.1.

Further, to better quantify mathematically the decrease in gap between the theoretical and simulated values of CDF as $K$ increases, we have derived the rate of convergence of the asymptotic minimum distribution to the corresponding Weibull distribution. We now give the rate of convergence for our case through the following theorem.

Theorem 2. The rate of convergence of $F_{\gamma_{\min }^{K}}(z)$ to the Weibull distribution is $\mathcal{O}\left(K^{-\mu^{-1}}+K^{-1}\right)$ where $\gamma_{\min }^{K}=\min \left\{\gamma_{1}, \cdots, \gamma_{K}\right\}$.

Proof. Please refer to Appendix B for the proof.

From this result, we observe that the rate of convergence depends on the length of the sequence $K$ and the source fading parameter $\mu$. The simulated and theoretical distribution are expected to be closer for large values of $K$. Further, the convergence will be faster for smaller values of $\mu$, the number of multi paths in the source to desired receiver link.

Using this asymptotic distribution, we can now determine the optimum $P_{s}$, when the number of PU-Rxs $M$, is moderate to large. To evaluate the CDF of $\gamma_{\min , p}:=$ $\lim _{M \rightarrow \infty} \min _{m}\left\{\frac{h_{m}}{\alpha_{m}} ; m=1, \cdots, M\right\}$ (to approximate (6b)), we now substitute $N=1, K=M,(\kappa, \mu, m, \bar{x})=$ $\left(\kappa_{p}, \mu_{p}, m_{p}, \bar{h}_{p}\right),\left(\kappa_{1}, \mu_{1}, m_{1}, \bar{x}_{1}\right)=\left(\kappa_{p, s}, \mu_{p, s}, m_{p, s}, \bar{\alpha}_{p, s}\right)$, $a_{K}=a_{M}=F_{\gamma}^{-1}\left(\frac{1}{M}\right)$ and $v=\mu_{p}$ in Theorem $1^{3}$. Hence, we have $(5 b)$ is equivalent to the following,

$$
1-\exp \left(-\left(\frac{\gamma_{0} P_{s}}{P_{p} a_{M}}\right)^{\mu_{p}}\right) \leq p_{0} .
$$

Further rearrangement of (10) gives,

$$
P_{s} \leq \frac{P_{p} a_{M}}{\gamma_{0}}\left[-\ln \left(1-p_{0}\right)\right]^{1 / \mu_{p}} .
$$

The largest $P_{s}$ that satisfies the above constraint is given by

$$
P_{s}^{+}=\frac{P_{p} a_{M}}{\gamma_{0}}\left[-\ln \left(1-p_{0}\right)\right]^{1 / \mu_{p}} .
$$

${ }^{3}$ Here, $F_{\gamma}(z)$ is evaluated using (??) for $N=1$. Even if we consider multiple primary interferers, note that Theorem 1 gives the asymptotic distribution of the minimum SIR for a case where the receiver suffers from the interference of $N$ other transmitters. Therefore, the theoretical framework developed is applicable for a much broader framework. However, when we consider $(N-1)$ primary interferers having known transmit powers, the expression of the outage probability will be different and we will not have a closed form expression for the secondary user's power allocation. Furthermore, in cells having large cell radius, the interference arising from other primary transmitters can be neglected due to the associated high path loss. 


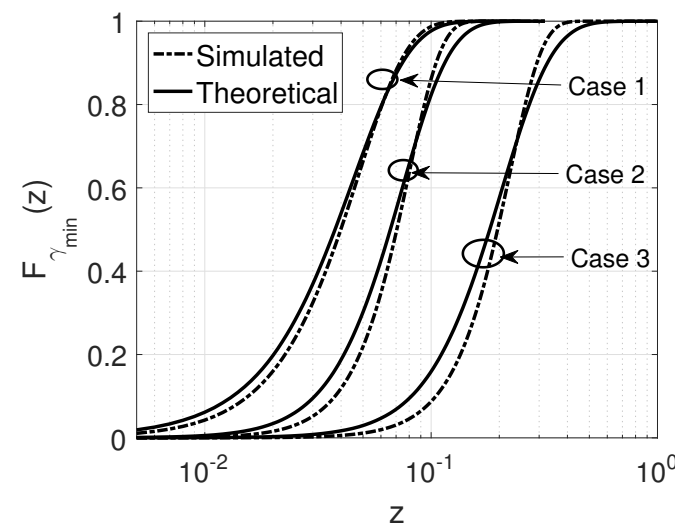

Fig. 1: CDF of $\gamma_{\min }^{K}$ (simulated) and $\gamma_{\min }$ (using (9)) for different fading scenarios.

Now, using (32) and (28d), the optimal $P_{s}$ for the SU-Tx power policy is given by

$$
\bar{P}_{s}=\min \left\{P_{s}^{+}, P_{s, \max }\right\} .
$$

To the best of our knowledge, there is no literature presenting the limiting distribution of the minimum SIR over a set of i.n.i.d RVs. However, if the i.n.i.d. RVs can be approximated by a set of i.i.d. RVs, then we can use our asymptotic results for the analysis. The effect of the i.i.d. assumption also depends on the way we replace the set of i.n.i.d. RVs by a set of i.i.d. RVs.

Now that we have derived the optimal SU-Tx power, we will analyze the impact of fading parameters on this power policy. From (31), we can observe that the optimum power at the SU-Tx, $\bar{P}_{s}$, is dependent on $P_{s}^{+}$given in (32). The variations in $P_{s}^{+}$are in turn governed by $P_{P}, \gamma_{0}, p_{0}, \mu_{p}$ and $a_{M}$.

Observation 1: From (32), it is plausible that an increase in either $P_{p}$ or $p_{0}$ or alternatively a decrease in $\gamma_{0}$ leads to an increase in $P_{s}^{+}$.

Note that, the variation in the SU transmit power $P_{s}^{+}$ with respect to the variations in the desired channel's fading conditions can be studied by analyzing the variations in $a_{M}$ and $\mu_{p}$. Since analyzing the effect of channel conditions on $a_{M}$ would mean analyzing the inverse CDF of SIR, which is difficult, we proceed by approximating each of the $\kappa-\mu$ shadowed RV by a Gamma RV. This approach is same as the approximations used in [45] Section III. Following analysis similar to [51], we can use stochastic ordering to make inferences about the approximate variation in $F_{\gamma}(z)$, with respect to the changes in $\kappa_{p}, \mu_{p}, m_{p}, \kappa_{p, s}, \mu_{p, s}$ and $m_{p, s}$. The corresponding observations are presented below. Since these observations can be easily derived by repeating steps similar to the derivation in [51] we do not repeat the details here.

Observation 2 : $P_{s}^{+}$increases upon increasing $\mu_{p}$ or $m_{p}$ or decreasing $\mu_{p, s}$ or $m_{p, s}$.
Observation $3: P_{s}^{+}$increases upon increasing $\kappa_{\boldsymbol{p}}$ if $\boldsymbol{m}_{\boldsymbol{p}}-\boldsymbol{\mu}_{\boldsymbol{p}} \geq \mathbf{0}$ and decreases otherwise. Alternatively, $P_{s}^{+}$ increases upon decreasing $\kappa_{p, s}$ if $\boldsymbol{m}_{p, s}-\mu_{p, s} \geq 0$ and decreases otherwise.

Thus Observation 2 and Observation 3 offers inferences on the variation of the maximum SU power $P_{s}^{+}$with respect to the changes in the source and interferer fading environment.

\section{B. Power allocation associated with the PU's statistical delay provision}

We assume that there is a queue at the PU-Tx, which is populated by some data arrival process at a constant rate of $R_{a}$ and has a stochastic service rate of $R_{p}$. In the context of $\mathrm{CR}$, the PU's delay requirement can be characterized either by the delay bound violation probability (DBVP) constraint or by the queue length bound violation probability constraint, as discussed in [22], [50]. Here, the probability of the PUs queuing-delay $D_{p}$ violating the threshold $D_{t h}$ is constrained to be less than $P_{t h}$, for a fixed PU-Tx transmit power. The transmit power policy at the SU-Tx can thus be mathematically formulated as follows:

$$
\begin{array}{ll} 
& \max P_{s}, \\
\text { s.t. } & \mathbb{P}\left\{D_{p} \geq D_{t h}\right\} \leq P_{t h}, \\
& P_{s} \leq P_{s, \max } .
\end{array}
$$

The authors of [22], [50] also considered constraint (14b) for their optimal power allocation problems. However, they maximized the ergodic rate of the secondary users in unicast scenarios. In the following steps, we will consider how the constraint in (14b) is evaluated for the multicast scenario.

The PU-Tx's DBVP can be rendered more tractable by a series of steps discussed in [50]. First of all, the DBVP can be approximated by the following relationship that relates the delay violation probability both to the arrival rate and to the delay exponent $\theta_{p}$ :

$$
\mathbb{P}\left\{D_{p} \geq D_{t h}\right\} \approx e^{-\theta_{p} E_{B} D_{t h}},
$$

where $E_{B}$ is the PU-Tx's effective bandwidth for the constant arrival process over a frame duration of $T_{f}$, which is given by

$$
E_{B}=\frac{1}{\theta_{p}} \log \left[\mathbb{E}\left(e^{\theta_{p} T_{f} B R_{a}}\right)\right],
$$

and the expectation is taken over the random channel fading coefficients. Therefore, the DBVP constraint can be reformulated in terms of a constraint on the delay exponent as

$$
\theta_{p} \geq-\frac{1}{E_{B} D_{t h}} \log \left(P_{t h}\right)
$$

Now, the effective capacity $E_{C}$ of the PU-Tx for a service rate of $R_{p}{ }^{4}$, is given by [22], [57]

$$
E_{C}=-\frac{1}{\theta_{p}} \log \left[\mathbb{E}\left(e^{-\theta_{p} T_{f} B R_{p}}\right)\right] \text {. }
$$

${ }^{4}$ Note that this is different from the arrival rate $R_{a}$ and is discussed in detail in [22] 
The expectation in (18) is also taken over the random channel fading coefficients. According to the theory of statistical queuing analysis, if the effective bandwidth $E_{B}$ is no higher than the effective capacity $E_{C}$, i.e., we have

$$
E_{B} \leq E_{C}
$$

then the constraint (14b) is equivalent to the following for a constant arrival process, [22], [50]

$$
E_{c} \geq T_{f} B R_{a}
$$

Thus the power allocation policy becomes

$$
\begin{array}{ll} 
& \max P_{s}, \\
\text { s.t. } & -\frac{1}{\theta_{p}} \log \left[\mathbb{E}\left(e^{-\theta_{p} T_{f} B R_{p}}\right)\right] \geq T_{f} B R_{a}, \\
& P_{s} \leq P_{s, \text { max }} .
\end{array}
$$

Now in a multicast case, the service rate $R_{p}$ would be limited by the link having the lowest SIR. Therefore, we have:

$R_{p}=\log _{2}\left(1+\min _{1 \leq m \leq M} \gamma_{m, p}\left(P_{s}\right)\right)=\log _{2}\left(1+\frac{P_{p}}{P_{s}} \gamma_{m i n, p}^{M}\right)$,

where $\gamma_{m i n, p}^{M}=\min _{1 \leq m \leq M} \frac{h_{m}}{\alpha_{m}}$. Upon further rearrangement, the constraint in (21b) can be rewritten as follows:

$$
\mathbb{E}\left[\left(1+\frac{P_{p}}{P_{s}} \gamma_{m i n, p}^{M}\right)^{-\xi}\right] \geq \exp \left(-\xi R_{a}\right)
$$

where $\xi=\frac{\theta_{p} T_{f} B}{\log 2}$. Here, increasing $P_{s}$ would decrease the effective capacity and hence the solution to the above optimization problem would correspond to the value of $P_{s}$, when (21b) is satisfied with equality. ${ }^{5}$ This solution can be readily found, for example using routines like FindRoot available in Mathematica. Note that the statistics of $R_{p}$ can be evaluated for large $M$ using the proposed asymptotic statistics of the minimum SIR. Hence, we make use of the asymptotic statistics from the previous sections to solve the power allocation problem in (21).

To analyze the power allocation policy we observe that when the constraint given in (23) is satisfied with equality, it can be rewritten as follows :

$$
\int_{0}^{\infty} \exp \left(-\left(\frac{\bar{P}_{s}\left(y^{-1 / \xi}-1\right)}{a_{M}}\right)^{\mu_{p}}\right) d y=\exp \left(-\xi R_{a}\right) .
$$

Based on (24) we can make observations similar to the ones in Section III.A of the revised manuscript. For example, the increase in $m_{p}$ results in an increase in $a_{M}$ which require an increase in the SU-Tx power $P_{s}$ for satisfying the equality in (24). Similar observations can be made with respect to other channel fading parameters as well.

\footnotetext{
${ }^{5}$ Similar arguments to obtain the SU power allocation policy is discussed in detail in [50].
}

\section{Power allocation under primary user secrecy outage con-} straint

In this section, we consider a system model very similar to the previous sub-section, except for the presence of a passive eavesdropper node $E$ trying to maliciously decode the information intended for the PU-Rxs. In such a scenario, the secrecy rate defined as the achievable rate of the legitimate receiver minus the rate overheard by the eavesdropper is considered as a reliable metric for evaluating the system's resilience to malicious attack [58]. Let, $f_{e}$ and $\theta_{e}$ represent the channel gains of the PU-Tx to $E$ and SU-TX to $E$ links, respectively. The SIR at the eavesdropper node $E$ is hence given by

$$
\gamma_{\text {eve }}=\frac{P_{p} f_{e}}{P_{s} \theta_{e}}=\frac{P_{p}}{P_{s}} \gamma_{e}
$$

The corresponding rate is hence $C_{\text {eve }}=\log _{2}\left(1+\gamma_{\text {eve }}\right)$. Thus, the PU's secrecy rate can be formulated as

$$
C_{s c y}=\log _{2}\left(1+\frac{P_{p}}{P_{s}} \gamma_{m i n, p}\right)-\log _{2}\left(1+\frac{P_{p}}{P_{s}} \gamma_{e}\right) .
$$

Note that here we compute the secrecy rate with respect to the ergodic multicast rate of the primary network. Hence, the probability of secrecy outage is given by

$$
\mathbb{P}\left(C_{s c y}<\delta_{s c y}\right)
$$

where $\delta_{s c y}$ represents the minimum secrecy rate. To ensure secure communication for the primary network in the CR scenario considered, the probability in (27) should remain small. Furthermore, note that the SU-Tx transmission creates interference for both the PU-Tx to PU-Rx link and PU-Tx to $E$ link. Hence, the power provided for the secondary network should also ensure that the probability of secrecy outage is sufficiently low. The secondary power allocation problem can thus be formulated as follows:

$$
\begin{aligned}
& \max P_{s} \\
\text { s.t. } & \mathbb{P}\left\{\min _{1 \leq m \leq M} \gamma_{m, p}\left(P_{s}\right) \leq \gamma_{0}\right\} \leq p_{0}^{\text {out }}, \\
& \mathbb{P}\left(C_{s c y}<\delta_{s c y}\right) \leq p_{0}^{s c y} \\
& P_{s} \leq P_{s, \text { max }} .
\end{aligned}
$$

Here, $p_{0}^{s c y}$ is the maximum affordable secrecy outage probability of the primary network and (28b) is the interference constraint of the primary network. The authors of [49], [59], [60] also consider secrecy constraints similar to (28c) for power allocation in different cognitive radio scenarios. However, neither of these contributions consider the case of multicast primary or secondary receivers. We have already established based on constraint (28b) that the maximum transmit power of the SU-Tx is limited by $P_{s} \leq \frac{P_{p} a_{M}}{\gamma_{0}}\left[-\ln \left(1-p_{0}\right)\right]^{1 / \mu_{p}}$. Next, let us consider the implication of constraint (28c), which can be re-written as follows:

$$
\mathbb{P}\left(\frac{P_{s}+P_{p} \gamma_{\min , p}}{P_{s}+P_{p} \gamma_{e}}-1 \leq 2^{\delta_{s c y}}-1\right) \leq p_{0}^{s c y} .
$$

Upon further re-arrangement the above expression can be equivalently expressed as

$$
\mathbb{P}\left(\frac{P_{p}\left(\gamma_{\min , p}-2^{\delta_{s c y}} \gamma_{e}\right)}{2^{\delta_{s c y}}-1} \leq P_{s}\right) \leq p_{0}^{s c y}
$$


From (30) it is clear that upon increasing $P_{s}$ the probability of secrecy outage increases. Hence, for maximizing $P_{s}$ the constraint in (28c) should be satisfied with equality. Let $\tilde{P}_{s}$ be the solution corresponding to equation (30) satisfied with equality. Thus, the optimal solution for the secondary power allocation problem is given by

$$
\bar{P}_{s}=\min \left\{P_{s}^{+}, \tilde{P}_{s}, P_{s, \max }\right\},
$$

where we have

$$
\begin{gathered}
P_{s}^{+}=\frac{P_{p} a_{M}}{\gamma_{0}}\left[-\ln \left(1-p_{0}\right)\right]^{1 / \mu_{p}} \quad \text { and } \\
\mathbb{P}\left(\frac{\tilde{P}_{s}+P_{p} \gamma_{\min , p}}{\tilde{P}_{s}+P_{p} \gamma_{e}} \leq 2^{\delta_{s c y}}\right)=p_{0}^{s c y} .
\end{gathered}
$$

From our statistical analysis of the distribution of the minimum of SIR RVs, $\gamma_{\min , p}$ follows the Weibull distribution. The $\mathrm{RV} \gamma_{e}$ is a ratio of two $\kappa-\mu$ shadowed RVs and solving (33) with the exact distribution of this ratio will be difficult. Hence, we approximate each of the $\kappa-\mu$ shadowed RV by a gamma RVs as justified in [41]. Thus, the distribution of $\gamma_{e}$ can be approximated by a Beta Prime distribution and the resultant expression (33) can be readily evaluated using common routines available in applications like Mathematica. Note that this would not be easy if we were to use the exact distribution of the minimum PU-SIR. Finally, observations similar to Observations $1-5$ of Section III.A can be derived for this case as well.

\section{ERGODIC MUTICAST RATE OF SECONDARY USERS}

For all the above optimization problems, once the optimum SU-Tx power $\bar{P}_{s}$ is determined, we can also obtain the ergodic multicast rate of the secondary users with EVT. The ergodic multicast rate of the secondary network is defined as [19], [61]

$$
C_{\text {sec }}=L \times \mathbb{E}\left[\log _{2}\left(1+\min _{1 \leq l \leq L} \gamma_{l, s}\right)\right]
$$

Substituting the expression for $\gamma_{l, s}$ from (2), we obtain

$$
C_{\text {sec }}=L \times \mathbb{E}\left[\log _{2}\left(1+\min _{1 \leq l \leq L} \frac{\bar{P}_{s} g_{l}}{P_{p} \beta_{l}}\right)\right] .
$$

Given that the CDF of the ratio of $\kappa-\mu$ shadowed RVs itself is complicated, it is a challenge to derive any simple expression for (35). Therefore, we propose the following theorem to evaluate the asymptotic ergodic multicast rate of SUs.

Theorem 3. Consider $K$ i.i.d. SIR RVs of the form

$$
\gamma_{k}=\frac{\left|b_{k}\right|^{2}}{\sum_{j=1}^{N}\left|c_{j, k}\right|^{2}},
$$

where $\left\{\left|b_{k}\right|^{2} ; 1 \leq k \leq K,\right\}$ are i.i.d. $\kappa-\mu$ shadowed $R V s$ with parameters $(\kappa, \mu, m, \bar{x})$ and $\left\{\left|c_{j, k}\right|^{2} ; 1 \leq j \leq N\right\}$ are i.n.i.d. $\kappa-\mu$ shadowed RVs, with parameters $\left(\kappa_{j}, \mu_{j}, m_{j}, \bar{x}_{j}\right)$ $\forall k$, for $j=1, . ., N$. If $\gamma_{\min }^{K}=\min \left(\gamma_{1}, \gamma_{2}, \ldots, \gamma_{K}\right)$, then

$$
\lim _{K \rightarrow \infty} \mathbb{E}\left[\log _{2}\left(1+\gamma_{\min }^{K}\right)\right]=\mathbb{E}\left[R_{\min }\right] \text {, }
$$

where $R_{\min }=\log _{2}\left(1+\gamma_{\min }\right)$ and $\gamma_{\min }$ is the asymptotic distribution of $\gamma_{\min }^{K}$ as given in Theorem (1).
Proof. The proof is very similar to the proof in [51, Section IV.2] and hence is omitted here.

The expectation in (34) can now be evaluated using the pdf of the Weibull RV, whose CDF is given in (9), after substituting $N=1, K=L,(\kappa, \mu, m, \bar{x}):=\left(\kappa_{s}, \mu_{s}, m_{s}, \bar{g}_{s}\right)$, $\left(\kappa_{1}, \mu_{1}, m_{1}, \bar{x}_{1}\right):=\left(\kappa_{s, p}, \mu_{s, p}, m_{s, p}, \bar{\beta}_{s, p}\right), a_{K}=a_{L}=$ $F_{\gamma}^{-1}\left(\frac{1}{L}\right)$ and $v=\mu_{s}$. The asymptotic minimum ergodic multicast rate of the secondary network is therefore given by

$$
\frac{C_{\text {sec }}}{L} \approx \int_{0}^{\infty} \log _{2}\left(1+\frac{P_{s} x}{P_{p}}\right) \frac{v x^{v-1}}{a_{L}^{v}} \exp \left(-\left(\frac{x}{a_{L}}\right)^{v}\right) d x .
$$

To analyze the above expression with respect to $a_{L}$, we again use the theory of stochastic ordering similar to [51]. Thus we have the following observations.

Observation $4: C_{s e c}$ increases upon increasing $\boldsymbol{m}_{\boldsymbol{s}}$ or decreasing $\boldsymbol{m}_{\boldsymbol{s}, \boldsymbol{p}}$ or $\boldsymbol{\mu}_{\boldsymbol{s}, \boldsymbol{p}}$.

Observation 5: $C_{\text {sec }}$ increases upon increasing $\boldsymbol{\kappa}_{\boldsymbol{s}}$ if $\boldsymbol{m}_{\boldsymbol{s}}-\boldsymbol{\mu}_{\boldsymbol{s}} \geq \mathbf{0}$ and decreases otherwise. Alternatively, $C_{s e c}$ increases upon decreasing $\kappa_{s, p}$ if $\boldsymbol{m}_{\boldsymbol{s}, \boldsymbol{p}}-\boldsymbol{\mu}_{\boldsymbol{s}, \boldsymbol{p}} \geq \mathbf{0}$ and decreases otherwise.

Observation 6 : Also, $C_{s e c}$ is directly proportional to $P_{s}^{+}$. Hence, variation in $C_{s e c}$ with respect to the variations in the fading channel of the primary network can be directly extended from Observation 2 and Observation 3.

\section{Numerical RESUlts AND Simulations}

In this section we present simulations to validate the results and observations. The PU-Tx's target rate is chosen to be $R_{0}=0.03 \mathrm{bps} / \mathrm{Hz}$ for all the simulations. This is to match the performance target for the operational long-term evolution (LTE) network, which requires the cell edge user throughput to be higher than $0.02 \mathrm{bps} / \mathrm{Hz} / \mathrm{cell} /$ user [19], [62], [63]. Similarly, all the results are generated for the choice of $P_{s, \max }=20 \mathrm{~dB}$. Here, Fig. 2 shows the SU-Tx power allocation for various combinations of PU-Tx power $P_{p}$ and PU-Rx outage constraint $p_{0}$ computed using (13). Furthermore, we have chosen $\left(\kappa_{p}=3, \mu_{p}=2, m_{p}=1\right),\left(\kappa_{p, s}=2, \mu_{p, s}=2, m_{p, s}=1\right)$, $\left(\kappa_{s}=2, \mu_{s}=2, m_{s}=1\right),\left(\kappa_{s, p}=3, \mu_{s, p}=3, m_{s, p}=1\right)$, $M=10$ and $L=10$ for generating Figs. $2-4$. The results indicate that the optimum SU-Tx power $\bar{P}_{s}$ increases upon increasing the PU-Tx power $P_{p}$. This is because, upon increasing $P_{p}$, the PU-Rxs become capable of handling a higher interference arriving from the SU-Tx at the same outage constraints. Furthermore, for constant $P_{p}, \bar{P}_{s}$ decreases with a reduction in $p_{0}$. This is because a reduction in $p_{0}$ results in stricter outage constraints at the PU-Rxs. In order to satisfy these stricter reliability conditions, the SU-Tx has to transmit at a lower power for reducing the interference at the PU-Rx. Note that the optimum transmit power $\bar{P}_{s}$ is always limited by $P_{s, \max }$. For the power allocation considered in Fig. 2, we show the simulated values of outage probabilities of both the primary and of the secondary receiver having lowest $\operatorname{SIR}\left(P_{p}^{\text {out }}\right.$ and $P_{s}^{\text {out }}$ ) in Fig. 3 and 4, respectively, for a threshold of $R_{0}=0.03$. Here, note that we are not constraining the outage probability of the secondary users in the allocation scheme 


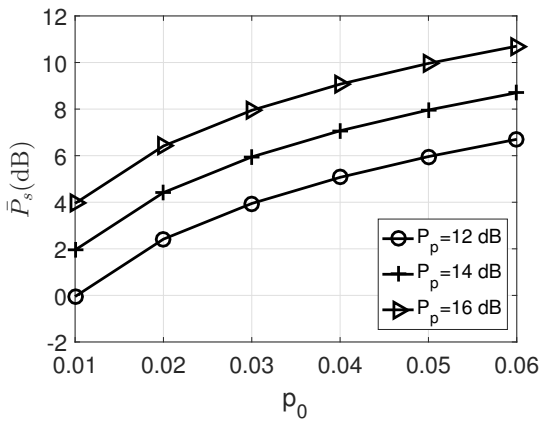

Fig. 2: $p_{0}$ vs $\bar{P}_{s}$ for $\mathrm{M}=10$ and $\mathrm{L}=10$.

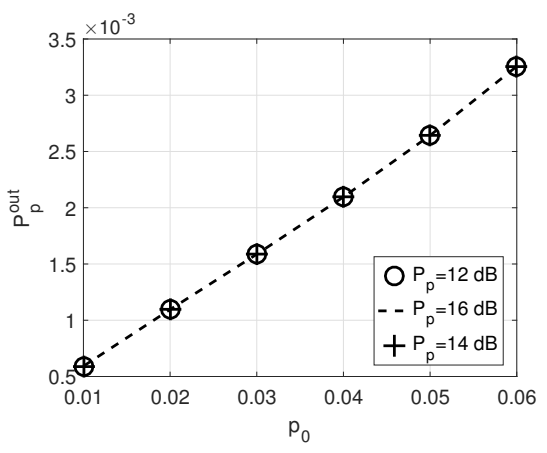

Fig. 3: $p_{o}$ vs $P_{p}^{\text {out }}$.

and hence the probability of outage of the secondary users may change with the channel conditions or system model.

Fig. 5 shows plots of $P_{p}$ versus $\bar{P}_{s}$ for different channel conditions to validate Observation 2. The channel parameters corresponding to the cases shown in the figure are given in Table III. From Cases 1,2 and 5 we can observe an increase in $\bar{P}_{s}$ with an increase in $\mu_{p}$ and $m_{p}$. Similarly, we can observe an decrease in $\bar{P}_{s}$ with an increase in $\mu_{p, s}$ and $m_{p, s}$ from cases 2,3 and 4. Next, Observation 3 is validated using simulations in Fig. 6. The fading channel parameters used for simulation are given in Table IV. According to Observation 3, variation in $\bar{P}_{s}$ with changes in $\kappa_{p}$ or $\kappa_{p, s}$ depends upon the sign of $\mu_{p}-m_{p}$ and $\mu_{p, s}-m_{p, s}$ respectively. We verify all such variations possible using cases 1-8 in Fig. 6. The above figures validate the claim that the proposed asymptotic results can be readily used to derive inferences on the system performance. Without the proposed simple distribution for the minimum SIR $\mathrm{RV}$, predicting the changes in the underlay CRN performance with respect to variations in channel fading conditions would have been non-trivial.

Next, we show power allocation results under our queuing delay constraints. The following parameter values were chosen for this simulation: $\left(\kappa_{p}=3, \mu_{p}=1, m_{p}=1\right)$, $\left(\kappa_{p, s}=2, \mu_{p, s}=2, m_{p, s}=1\right), T_{f}=2 \mathrm{~ms}, B=10^{5}$ Hz, $R_{a}=1.5$ and $P_{s, \max }=15 \mathrm{~dB}$. Here, Fig. 7 shows the variation in SU-Tx power (computed using (23)) upon increasing the delay exponent $\theta_{p}$. Note that a smaller value of $\theta_{p}$ corresponds to looser delay constraint and hence allows SU-Tx to transmit at higher power as compared to larger values of $\theta_{p}$. Fig. 8 shows the corresponding values of effective capacity. Note that constraint (21b) is satisfied in all the

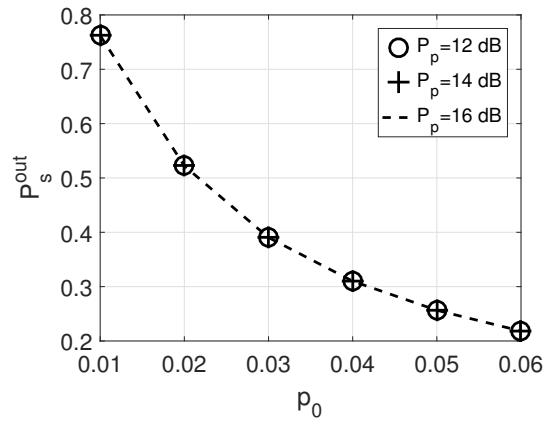

Fig. 4: $p_{0}$ vs $P_{s}^{\text {out }}$.

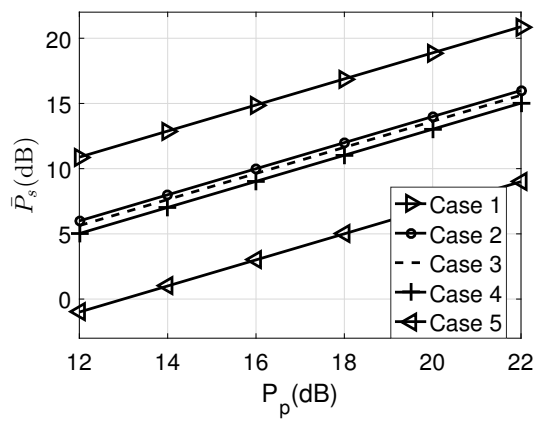

Fig. 5: $P_{p}$ vs $\bar{P}_{s}$ for $\mathrm{M}=20$.

cases. Similarly, we show power allocation results (computed using (31)) for the secrecy constraints in Fig. 9 to 10. Here, $\left(\kappa_{p, e}, \mu_{p, e}, m_{p, e}\right)$ and $\left(\kappa_{s, e}, \mu_{s, e}, m_{s, e}\right)$ represent the $\kappa-\mu$ shadowed parameters of RVs $f_{e}$ and $\theta_{e}$ respectively. Furthermore, the following parameters were chosen for simulation: $\left(\kappa_{p}=3, \mu_{p}=2, m_{p}=3\right),\left(\kappa_{p, s}=5, \mu_{p, s}=3, m_{p, s}=3\right)$, $\left(\kappa_{p, e}=1, \mu_{p, e}=0.01, m_{p, e}=0.1\right),\left(\kappa_{s, e}=1, \mu_{s, e}=\right.$ $\left.2, m_{s, e}=2\right), \delta^{s y c}=1, p_{0}=0.01$ and $\gamma_{0}=0.02$. Here, Fig. 9 and 10 shows the power allocation and the corresponding value of secrecy outage for different values of $p_{0}^{s y c}$, respectively.

Next, in Figs. 11 we compare the simulated and theoretical values of the ergodic multicast rate of secondary users, for $P_{p}=14 \mathrm{~dB}$. Fig. 11 shows the variation in $C_{s e c} / L$ with respect to variation in number of primary users $M$, for two different values of $p_{0}$. The fading channel parameters used for simulation are as follows : $\left(\kappa_{p}=2, \mu_{p}=3, m_{p}=1\right)$, $\left(\kappa_{p, s}=2, \mu_{p, s}=2, m_{p, s}=1\right),\left(\kappa_{s}=2, \mu_{s}=2, m_{s}=1\right)$, $\left(\kappa_{s, p}=3, \mu_{s, p}=3, m_{s, p}=1\right)$. Observations $4-5$ can also be verified via simulation. However, these are not provided here due to space constraints.

\section{SUMMARY}

To summarize, we make use of tools from EVT to characterize the asymptotic distribution of the minimum of the ratio of $\kappa-\mu$ shadowed random variables and hence derive a simple expression for the distribution of the minimum SIR of PU/SU in a multicast CR environment. We also derive the rate of convergence of the actual distribution of the minimum SIR to the derived distribution. These results are further used to find the optimal SU power allocation and the ergodic multicast rate of SUs under different QoS constraints. 


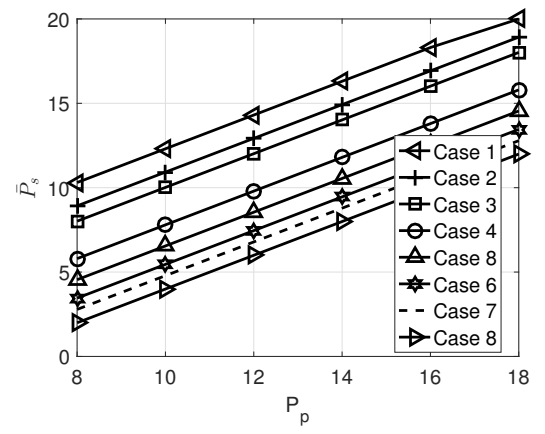

Fig. 6: $P_{p}$ vs $\bar{P}_{s}$ for $\mathrm{M}=20$.

\begin{tabular}{|c|c|c|c|c|c|c|}
\hline Case \# & $\kappa_{p}$ & $\mu_{p}$ & $m_{p}$ & $\kappa_{p, s}$ & $\mu_{p, s}$ & $m_{p, s}$ \\
\hline 1 & 3 & 2 & 1 & 2 & 1 & 1 \\
\hline 2 & 3 & 1 & 1 & 2 & 1 & 1 \\
\hline 3 & 3 & 1 & 1 & 2 & 20 & 1 \\
\hline 4 & 3 & 1 & 1 & 2 & 1 & 10 \\
\hline 5 & 3 & 1 & 0.1 & 2 & 1 & 1 \\
\hline
\end{tabular}

TABLE III: Channel parameters used for simulation of Fig.5.

Assuming all the links are undergoing $\kappa-\mu$ shadowed fading, we have used results from stochastic ordering to analyze the impact of various channel parameters on the SU performance and have derivied analytical observations for the case of SU power allocation subject to interference constraints at the primary receivers. Conclusive observations were also made for the cases of queuing delay constraints and secrecy outage constraints at the primary receivers. Owing to space constraints these observations are not included in the following table: 6

\section{APPENDIX A}

PROOF FOR THEOREM 1

We know that $\gamma_{\text {min }}^{K}=\min \left\{\gamma_{1}, \cdots, \gamma_{K}\right\}=$ $-\max \left\{-\gamma_{1}, \cdots,-\gamma_{K}\right\}$. Now, if we derive the asymptotic distribution of the maximum of $K$ i.i.d. RVs $\hat{\gamma}_{\max }^{K}=\max \left\{\hat{\gamma}_{1}, \cdots, \hat{\gamma}_{K}\right\}$ where $\hat{\gamma}_{l}=-\gamma_{l} ; l=1, \cdots, K$ and $\hat{\gamma}_{l} \sim F_{\hat{\gamma}}(z)=1-F_{\gamma}(-z)$ then we can also derive the asymptotic distribution of $\gamma_{\mathrm{min}}^{K}$. Now, we invoke the following theorem to derive the limiting distribution of $\hat{\gamma}_{\max }^{K}$.

Theorem 4. Fisher-Tippet Theorem, Limit Laws for Maxima: Let $z_{1}, z_{2}, \cdots, z_{K}$ be a sequence of $K$ i.i.d. RVs and $M_{K}=$ $\max \left\{z_{1}, z_{2}, \cdots, z_{K}\right\}$; if $\exists$ constants that obey $a_{K}>0$ and $b_{K} \in \mathbb{R}$ and some non-degenerate $C D F G_{\nu}$ so that when $K \rightarrow \infty$ we have,

$$
a_{K}^{-1}\left(M_{K}-b_{K}\right) \stackrel{D}{\rightarrow} G_{\nu},
$$

where $\stackrel{D}{\rightarrow}$ denotes convergence in distribution. Then the CDF $G_{v}$ is one of the three CDFs:

Frechet [64]: $\Lambda_{1}(z):= \begin{cases}0, & z \leq 0 \\ \exp \left(-z^{-v}\right), & z>0,\end{cases}$

Reversed Weibull [64] : $\quad \Lambda_{2}(z) \quad:=$ $\left\{\exp \left(-(-z)^{v}\right), \quad z \leq 0\right.$,

1, $\quad z>0$

Gumbel [64]: $\Lambda_{3}(z):=\exp (-\exp (-z)), \quad z \in \mathbb{R}$.

${ }^{6}$ Here, $\uparrow$ and $\downarrow$ are used to represent increase and decrease respectively.

\begin{tabular}{|c|c|c|c|c|c|c|}
\hline Case \# & $\kappa_{p}$ & $\mu_{p}$ & $m_{p}$ & $\kappa_{p, s}$ & $\mu_{p, s}$ & $m_{p, s}$ \\
\hline 1 & 3 & 2 & 1 & 2 & 1 & 1 \\
\hline 2 & 10 & 2 & 1 & 2 & 1 & 1 \\
\hline 3 & 3 & 1 & 10 & 2 & 1 & 2 \\
\hline 4 & 3 & 1 & 10 & 10 & 1 & 2 \\
\hline 5 & 10 & 1 & 2 & 2 & 1 & 1 \\
\hline 6 & 3 & 1 & 2 & 2 & 1 & 1 \\
\hline 7 & 3 & 1 & 1 & 30 & 2 & 1 \\
\hline 8 & 3 & 1 & 1 & 2 & 2 & 1 \\
\hline
\end{tabular}

TABLE IV: Channel parameters used for simulation of Fig.6

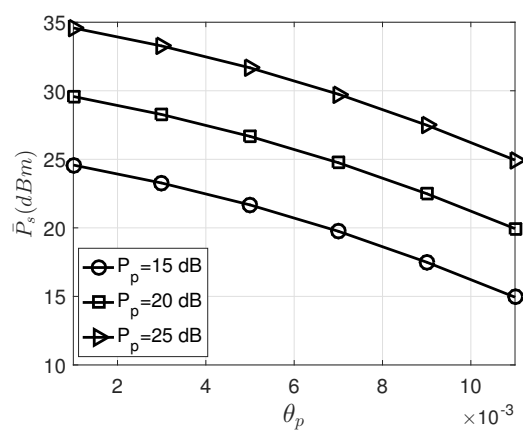

Fig. 7: $\theta_{p}$ vs $\bar{P}_{s}$ for $M=20$.

Proof. Please refer to [65] for the proof.

To determine the limiting distribution among these three, we first have to define the Maximum Domain of Attraction $(M D A)$.

Definition 1. Maximum Domain of Attraction [65]: The CDF $F$ of i.i.d. RVs $z_{1}, \cdots, z_{K}$ belongs to the $M D A$ of the extreme value distribution (EVD) $G_{v}$, if and only if $\exists$ the constants obeying $a_{K}>0$ and $b_{K} \in \mathbb{R}$, so that (39) holds.

Lemma 1. Let $F$ be a distribution function and $x^{*}:=\sup \{x$ : $F(x)<1\}$. Let us assume that $F^{\prime \prime}(x)$ exists and $F^{\prime}(x)$ is positive for all $x$ in some left neighborhood of $x^{*}$. If

$$
\lim _{x \rightarrow x^{*}} \frac{\left(x^{*}-x\right) f(x)}{1-F(x)}=v ; v>0,
$$

then $F($.$) belongs to the MDA of the reversed Weibull distri-$ bution.

Proof. Please refer to [65] for the proof.

Now, if we show that the $\mathrm{CDF} F_{\hat{\gamma}}(z)$ satisfies the relationship in (40), then from the definition of the $M D A$ of an EVD, we may conclude that there exists $a_{K}$ and $b_{K}$ satisfying (39). A choice for the corresponding constants of the reversed Weibull distribution is given in [65] as $b_{K}=0$ and $a_{K}=-F_{\hat{\gamma}}^{-1}\left(1-K^{-1}\right)$.

Theorem 5. The CDF $F_{\hat{\gamma}}(z)$ is in the MDA of the reversed Weibull distribution.

Proof. Here we would have to evaluate the following limit :

$$
\lim _{z \rightarrow 0} \frac{(-z) f_{\hat{\gamma}}(z)}{1-F_{\hat{\gamma}}(z)} .
$$

Now, by exploiting the properties of the transformation of RVs, we have $f_{\hat{\gamma}}(z)=f_{\gamma}(-z)$ and $F_{\hat{\gamma}}(z)=1-F_{\gamma}(-z)$. Thus, (41) 


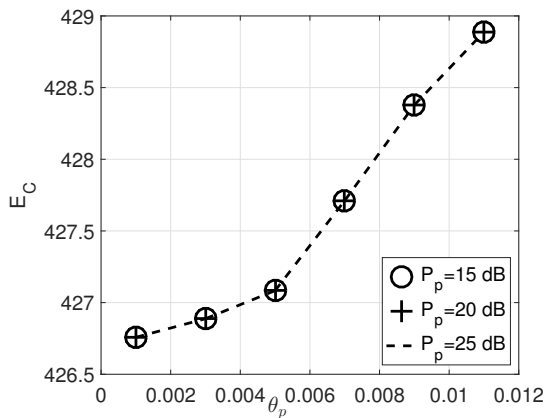

Fig. 8: $\theta_{p}$ vs $E_{C}$ for minimum SIR primary user.

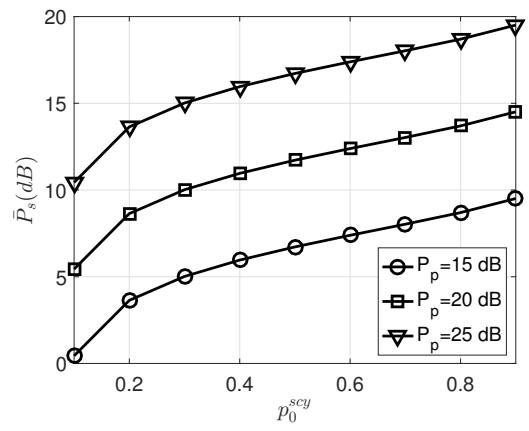

Fig. 9: $p_{o}^{s c y}$ vs $\bar{P}_{s}$ for $M=20$.

can be evaluated as

$$
\lim _{z \rightarrow 0} \frac{(-z) f_{\gamma}(-z)}{F_{\gamma}(-z)}
$$

The pdf $f_{\gamma}(z)$ is given by (43), where $K_{2}=\frac{\theta^{\left(m+\sum_{i=1}^{N} \mu_{i}\right)} \Gamma\left[\mu+\sum_{i=1}^{N} \mu_{i}\right]}{\lambda^{m} \Gamma[\mu] \Gamma\left[\sum_{i=1}^{N} \mu_{i}\right] \prod_{i=1}^{N} \theta_{i}^{\mu_{i}-m_{i}} \lambda_{i}^{m_{i}}} ;$

Similarly, from [46, Eqn. (6)], we have (44) where $K_{1}=\frac{\Gamma\left[\sum_{1=1}^{N} \mu_{i}+\mu\right]\left(\prod_{i=1}^{N} \theta_{i}^{-\left(\mu_{i}-m_{i}\right)} \lambda_{i}^{-m_{i}}\right) \theta^{\sum_{i=1}^{N} \mu_{i}+m}}{\Gamma\left[\sum_{1=1}^{N} \mu_{i}+1\right] z^{\sum_{i=1}^{N} \mu_{i}} \lambda^{m} \Gamma[\mu]}$.

We now have to evaluate the limit of $F_{\gamma}(-z)$ in the denominator of (42), and the above expression of the CDF is available in the $[1-\mathrm{CCDF}]$ form. For ease of further analysis we reformulate the $\mathrm{CDF}$ as given in (45). ${ }^{7}$

Now, we can make use of the following properties of the limits to proceed with the evaluation of (42):

- $\lim _{x \rightarrow a}[f(x) g(x)]=\lim _{x \rightarrow a} f(x) . \lim _{x \rightarrow a} g(x)$

- $\lim _{x \rightarrow a} \frac{f(x)}{g(x)}=\frac{\lim _{x \rightarrow a} f(x)}{\lim _{x \rightarrow a} g(x)}$, if $\lim _{x \rightarrow a} g(x) \neq 0$.

We first consider the ratio without the $E_{D}($.$) terms. Here,$ we have

\footnotetext{
${ }^{7}$ This proof is not included in this paper since it is derived by repeating steps very similar to the derivation of CCDF in [46].
}

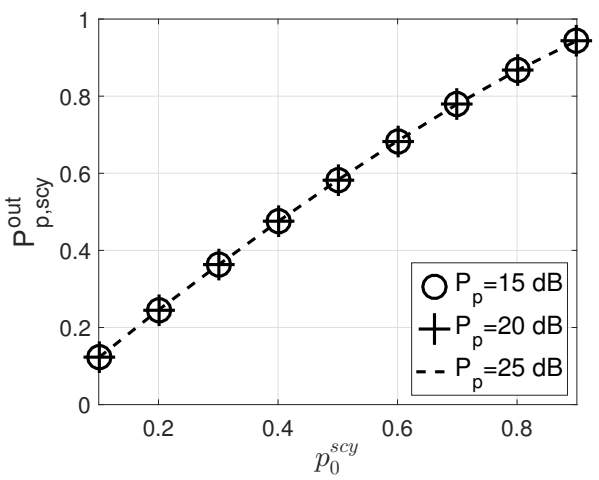

Fig. 10: $p_{o}^{s c y}$ vs $P_{p, s c y}^{o u t}$ for minimum SIR primary user.

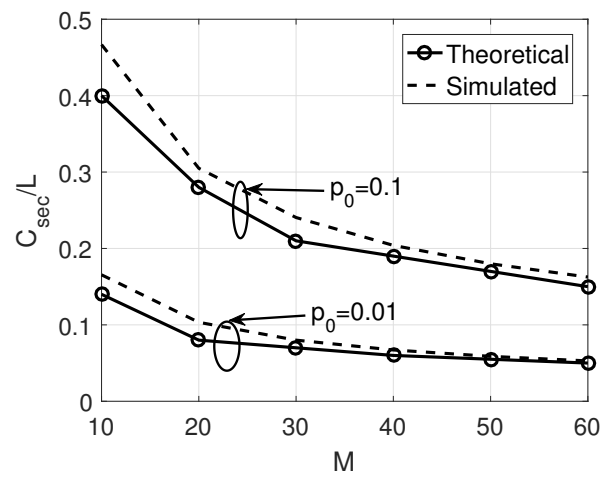

Fig. 11: $\mathrm{M}$ vs $C_{s e c} / L$.

$$
\begin{aligned}
& (-z)^{\mu} \theta^{m+\sum_{i=1}^{N} \mu_{i}} \Gamma\left[\mu+\sum_{i=1}^{N} \mu_{i}\right] \theta_{1}^{\mu+\sum_{i=1}^{N} \mu_{i}} \\
& \lim _{z \rightarrow 0} \frac{\overline{\lambda^{m} \Gamma[\mu] \Gamma\left[\sum_{i=1}^{N} \mu_{i}\right] \prod_{i=1}^{N} \theta_{i}^{\mu_{i}-m_{i}} \lambda_{i}^{m_{i}}\left(\theta-z \theta_{1}\right)^{\mu+\left[\sum_{i=1}^{N} \mu_{i}\right.}}}{(-z)^{\mu} \theta^{m+\sum_{i=1}^{N} \mu_{i}} \Gamma\left[\mu+\sum_{i=1}^{N} \mu_{i}\right] \theta_{1}^{\mu+\sum_{i=1}^{N} \mu_{i}}} \\
& \lambda^{m} \Gamma[\mu+1] \Gamma\left[\sum_{i=1}^{N} \mu_{i}\right] \prod_{i=1}^{N} \theta_{i}^{\mu_{i}-m_{i}} \lambda_{i}^{m_{i}}\left(\theta-z \theta_{1}\right){ }^{\mu+\left[\sum_{i=1}^{N} \mu_{i}\right.} \\
& =\lim _{z \rightarrow 0} \frac{\Gamma[\mu+1]}{\Gamma[\mu]}=\mu .
\end{aligned}
$$

Now, if we analyze the $E_{D}($.$) term in the numerator, we$ have

$$
\begin{aligned}
& { }_{(1)}^{(1)} E_{D}^{(2 N)}\left[\mu+\sum_{i=1}^{N} \mu_{i}, m, \mu_{2}-m_{2}, \cdots, \mu_{N}-m_{N}, m_{1},\right. \\
& \cdots, m_{N}, \mu, \sum_{i=1}^{N} \mu_{i}, \frac{-z \theta_{1}(\lambda-\theta)}{\lambda\left(\theta-z \theta_{1}\right)}, \frac{\theta\left(\theta_{2}-\theta_{1}\right)}{\theta_{2}\left(\theta-z \theta_{1}\right)}, \cdots, \\
& \left.\frac{\theta\left(\theta_{N}-\theta_{1}\right)}{\theta_{N}\left(\theta-z \theta_{1}\right)}, \frac{\theta\left(\lambda_{1}-\theta_{1}\right)}{\lambda_{1}\left(\theta-z \theta_{1}\right)}, \cdots, \frac{\theta\left(\lambda_{N}-\theta_{1}\right)}{\lambda_{N}\left(\theta-z \theta_{1}\right)}\right],
\end{aligned}
$$

and the $E_{D}($.$) function has the following series expansion:$

${ }_{(1)}^{(k)} E_{D}^{(n)}\left(a, b_{1}, \cdots, b_{n} ; c, c^{\prime} ; x_{1}, \cdots, x_{n}\right)=$

$$
\sum_{i_{1} \cdots i_{n}=0}^{\infty} \frac{(a)_{i_{1}+\cdots+i_{n}}\left(b_{1}\right)_{\left(i_{1}\right)} \cdots\left(b_{n}\right)_{\left(i_{n}\right)} x_{1}^{i_{1}} \cdots x_{n}^{i_{n}}}{(c)_{\left(i_{1}+\cdots+i_{k}\right)}\left(c^{\prime}\right)_{\left(i_{k+1}+\cdots+i_{n}\right)} i_{1} ! \cdots i_{n} !} .
$$




$$
\begin{aligned}
& f_{\gamma}(z)=K_{2} z^{-\left(1+\sum_{i=1}^{N} \mu_{i}\right)}\left(1+\frac{\theta}{z \theta_{1}}\right)^{-\left(\mu+\sum_{i=1}^{N} \mu_{i}\right)} \times \times_{(1)}^{(1)} E_{D}^{(2 N)}\left[\mu+\sum_{i=1}^{N} \mu_{i}, m, \mu_{2}-m_{2}, \cdots, \mu_{N}-m_{N}, m_{1}, \cdots, m_{N}\right. \\
& \left.\mu, \sum_{i=1}^{N} \mu_{i}, \frac{z \theta_{1}(\lambda-\theta)}{\lambda\left(\theta+z \theta_{1}\right)}, \frac{\theta\left(\theta_{2}-\theta_{1}\right)}{\theta_{2}\left(\theta+z \theta_{1}\right)}, \cdots, \frac{\theta\left(\theta_{N}-\theta_{1}\right)}{\theta_{N}\left(\theta+z \theta_{1}\right)}, \frac{\theta\left(\lambda_{1}-\theta_{1}\right)}{\lambda_{1}\left(\theta+z \theta_{1}\right)}, \cdots, \frac{\theta\left(\lambda_{N}-\theta_{1}\right)}{\lambda_{N}\left(\theta+z \theta_{1}\right)}\right] . \\
& F_{\gamma}(z)=1-K_{1}\left(\frac{z \theta_{1}}{\theta+z \theta_{1}}\right)^{\sum_{i=1}^{N} \mu_{i}+\mu} \times \times_{(1)}^{(1)} E_{D}^{(2 N+1)}\left[\sum_{i=1}^{N} \mu_{i}+\mu, m, 1, \mu_{2}-m_{2}, \cdots, \mu_{N}-m_{N}, m_{1}, \cdots, m_{N}, \mu, 1+\sum_{i=1}^{N} \mu_{i}\right. \\
& \left.\frac{(\lambda-\theta) z \theta_{1}}{\left(\theta+z \theta_{1}\right) \lambda}, \frac{\theta}{\theta+z \theta_{1}}, \frac{\theta \theta_{2}-\theta \theta_{1}}{\theta_{2}\left(\theta+z \theta_{1}\right)}, \cdots, \frac{\theta \theta_{N}-\theta \theta_{1}}{\theta_{N}\left(\theta+z \theta_{1}\right)}, \frac{\theta \lambda_{1}-\theta \theta_{1}}{\lambda_{1}\left(\theta+z \theta_{1}\right)}, \cdots, \frac{\theta \lambda_{N}-\theta \theta_{1}}{\lambda_{N}\left(\theta+z \theta_{1}\right)}\right] . \\
& F_{\gamma}(z)=\frac{z^{-\sum_{i=1}^{N} \mu_{i}} \theta^{\sum_{i=1}^{N} \mu_{i}+m} \Gamma\left[\sum_{i=1}^{N} \mu_{i}+\mu\right] \lambda^{-m}}{\Gamma[\mu+1] \Gamma\left[\sum_{i=1}^{N} \mu_{i}\right] \prod_{i=1}^{N} \theta_{i}^{\mu_{i}-m_{i}} \lambda_{i}^{m_{i}}}\left(\frac{\theta_{1} z}{\theta+\theta_{1} z}\right)^{\mu+\sum_{i=1}^{N} \mu_{i}}{ }_{(1)}^{(2)} E_{D}^{2 N+1}\left[\mu+\sum_{i=1}^{N} \mu_{i}, 1, m, \mu_{2}-m_{2}, \cdots\right. \\
& \left.\mu_{N}-m_{N}, m_{1}, \cdots, m_{N}, \mu+1, \sum_{i=1}^{N} \mu_{i}, \frac{\theta_{1} z}{\theta+\theta_{1} z}, \frac{\theta(\lambda-\theta) z}{\lambda\left(\theta+\theta_{1} z\right)}, \frac{\theta\left(\theta_{2}-\theta_{1}\right)}{\theta_{2}\left(\theta+\theta_{1} z\right)}, \cdots, \frac{\theta\left(\theta_{N}-\theta_{1}\right)}{\theta_{N}\left(\theta+\theta_{1} z\right)}, \frac{\theta\left(\lambda_{1}-\theta_{1}\right)}{\lambda_{1}\left(\theta+\theta_{1} z\right)}, \cdots, \frac{\theta\left(\lambda_{N}-\theta_{1}\right)}{\lambda_{N}\left(\theta+\theta_{1} z\right)}\right]
\end{aligned}
$$

\begin{tabular}{|c|c|c|}
\hline $\begin{array}{c}\text { Increase in } \\
\text { system parameter }\end{array}$ & $\begin{array}{c}\text { Optimum } \\
\text { SU-Tx power }\left(\bar{P}_{s}\right)\end{array}$ & $\begin{array}{c}\text { Ergodic MC rate } \\
\text { of SU }\left(C_{s e c} / L\right)\end{array}$ \\
\hline$P_{p}$ & $\uparrow$ & $\uparrow$ \\
\hline$p_{0}$ & $\uparrow$ & $\uparrow$ \\
\hline$\gamma_{0}$ & $\downarrow$ & $\downarrow$ \\
\hline$L$ & $\downarrow$ & $\downarrow$ \\
\hline$\kappa_{p}$, if $m_{p}-\mu_{p}>0$ & - & $\downarrow$ \\
\hline$\kappa_{p}$, if $m_{p}-\mu_{p}<0$ & $\uparrow$ & $\uparrow$ \\
\hline$\mu_{p}$ & $\uparrow$ & $\uparrow$ \\
\hline$m_{p}$ & $\uparrow$ & $\uparrow$ \\
\hline$\kappa_{p, s}$, if $m_{p, s}-\mu_{p, s}>0$ & $\downarrow$ & $\downarrow$ \\
\hline$\kappa_{p, s}$, if $m_{p, s}-\mu_{p, s}<0$ & $\uparrow$ & $\uparrow$ \\
\hline$\mu_{p, s}$ & $\downarrow$ & $\downarrow$ \\
\hline$m_{p, s}$ & $\downarrow$ & $\uparrow$ \\
\hline$\kappa_{s}$, if $m_{s}-\mu_{s}>0$ & - & $\downarrow$ \\
\hline$\kappa_{s}$, if $m_{s}-\mu_{s}<0$ & - & $\uparrow$ \\
\hline$\mu_{s}$ & - & $\uparrow$ \\
\hline$m_{s}$ & - & $\downarrow$ \\
\hline$\kappa_{s, p}$, if $m_{s, p}-\mu_{s, p}>0$ & - & $\uparrow$ \\
\hline$\kappa_{s, p}$, if $m_{s, p}-\mu_{s, p}<0$ & - & $\downarrow$ \\
\hline$\mu_{s, p}$ & - & $\downarrow$ \\
\hline$m_{s, p}$ & - & $\downarrow$ \\
\hline
\end{tabular}

TABLE V: Table of variation in $\bar{P}_{s}$ and $C_{s e c} / L$ with increase in different system parameters

Thus, (47) can be expanded as

$$
\begin{aligned}
\sum_{p_{1}, \cdots, p_{2 N}=0}^{\infty} \frac{\left(\mu+\sum_{i=1}^{N} \mu_{i}\right)_{p_{1}+\cdots+p_{2 N}}(m)_{p_{1}}\left(\mu_{2}-m_{2}\right)_{p_{2}} \cdots}{(\mu)_{p_{1}}} \\
\frac{\left(\mu_{N}-m_{N}\right)_{p_{N}}\left(m_{1}\right)_{p_{N+1}} \cdots\left(m_{N}\right)_{p_{2 N}}}{\left(\sum_{i=1}^{N} \mu_{i}\right)_{p_{2}+\cdots+p_{2 N}}} \times \prod_{i=1}^{2 N} \frac{x_{i}^{p_{i}}}{p_{i} !},
\end{aligned}
$$

where $x_{1}=\frac{-z \theta_{1}(\lambda-\theta)}{\lambda\left(\theta-z \theta_{1}\right)}, x_{i}=\frac{\theta\left(\theta_{i}-\theta_{1}\right)}{\theta_{i}\left(\theta-z \theta_{1}\right)} ; i=2 \cdots N$ and $x_{i}=\frac{\theta\left(\lambda_{i}-\theta_{1}\right)}{\lambda_{i}\left(\theta-z \theta_{1}\right)} ; i=N+1 \cdots 2 N$. Note that, for $p_{1} \neq 0$, we have $\lim _{z \rightarrow 0} x_{1}=0$. Hence, at $\lim z \rightarrow 0$, only the terms corresponding to $p_{1}=0$ will remain with $x_{i} ; i=2, \cdots, 2 N$ evaluated at $z \rightarrow 0$. Similarly, if we now consider the $E_{D}($. term in the denominator (from the CDF expression), it has the following series expansion :

$$
\begin{gathered}
\sum_{p_{1}, \cdots, p_{2 N+1}=0}^{\infty} \frac{\left(\mu+\sum_{i=1}^{N} \mu_{i}\right)_{p_{1}+\cdots+p_{2 N}}(1)_{p_{1}}(m)_{p_{2}}\left(\mu_{2}-m_{2}\right)_{p_{2}}}{(\mu+1)_{p_{1}+p_{2}}} \\
\times \frac{\cdots\left(\mu_{N}-m_{N}\right)_{p_{N}}\left(m_{1}\right)_{p_{N+1}} \cdots\left(m_{N}\right)_{p_{2 N}}}{\left(\sum_{i=1}^{N} \mu_{i}\right)_{p_{3}+\cdots+p_{2 N}}^{2 N+1} \frac{x_{i}^{p_{i}}}{p_{i} !}}
\end{gathered}
$$

where $x_{1}=\frac{-z \theta_{1}}{\left(\theta-z \theta_{1}\right)}, x_{2}=\frac{\theta(\lambda-\theta)(-z)}{\lambda\left(\theta-\theta_{1} z\right)} x_{i}=\frac{\theta\left(\theta_{i}-\theta_{1}\right)}{\theta_{i}\left(\theta-z \theta_{1}\right)} ; i=$ $3 \cdots N+1$ and $x_{i}=\frac{\theta\left(\lambda_{i}-\theta_{1}\right)}{\lambda_{i}\left(\theta-z \theta_{1}\right)} ; i=N+2 \cdots 2 N+1$. Note that whenever $p_{1} \neq 0, p_{2} \neq 0, \lim _{z \rightarrow 0} x_{1}=0$ and $\lim _{z \rightarrow 0} x_{2}=0$, respectively. Hence, at $\lim z \rightarrow 0$, only the

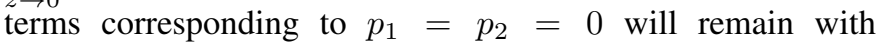
$x_{i} ; i=3, \cdots, 2 N+1$ evaluated at $z \rightarrow 0$. Now, note that this set of remaining terms is the same for both the $E_{D}$ terms in the numerator as well as the denominator. Hence, the ratio of these terms evaluates to one. Thus, we have

$$
\lim _{z \rightarrow 0} \frac{(-z) f_{\gamma}(-z)}{F_{\gamma}(-z)}=\lim _{z \rightarrow 0} \frac{(-z) f_{\hat{\gamma}}(z)}{1-F_{\hat{\gamma}}(z)}=\mu .
$$


Now we know that the asymptotic distribution of $\hat{\gamma}_{\max }^{K}$ is a reversed Weibull distribution, hence we conclude that the asymptotic distribution of the minimum of $K \operatorname{SIR} \operatorname{RVs}\left(\gamma_{\min }^{K}\right)$ is a Weibull distribution with shape parameter $v=\mu$ and the shape parameter $a_{K}$ as given in (9).

\section{APPENDIX B}

\section{DERIVATION OF RATE OF CONVERGENCE}

To prove the result in Theorem 2, we first define the $\delta$ neighborhood of generalized pareto distribution (GPD) for a Weibull RV. Let the $\delta$-neighbourhood be denoted by $Q_{2}(\delta)$ and the GPD for a Weibull RV be denoted by $W_{\{2, \nu\}}$. The Extreme Value Distributions (EVDs) lies in the $\delta$ neighbourhood of one of three GPD $W_{\{i, \nu\}} ; i=1,2,3$ with $\delta=1$.

Definition 2. $\delta$-neighborhood $Q_{2}(\delta)$ of the GPD $W_{\{2, \nu\}}$ [66] is defined as $Q_{2}(\delta):=\{\mathrm{F}: \omega(F)<\infty$ and $F$ has a density $f$ on $\left[z_{0}, \omega(F)\right]$ for some $z_{0}<\omega(F)$ such that for some shape parameter $\nu>0$ and some scale parameter $a>0$ on $\left[z_{0}, \omega(F)\right]$, we have,

$f(z)=\frac{1}{a} W_{2, \nu}^{\prime}\left(\frac{z-\omega(F)}{a}\right)\left(1+O\left(\left(1-W_{2, \nu}(z-\omega(F))\right)^{\delta}\right)\right\}$,

where $\omega(F):=\sup \{z \in \mathbb{R}: F(z)<1\}$. In fact the GPD for the Weibull distribution is defined in [66] as $W_{2, \nu}=1-(-z)^{\nu} ;-1 \leq z \leq 0$ and using this, (52) can be rewritten as

$$
f(z)=\frac{\nu}{a}\left(\frac{-z+\omega(F)}{a}\right)^{\nu-1}\left(1+O\left(\left((-z+\omega(F))^{\nu}\right)^{\delta}\right)\right) .
$$

This definition says that, if a PDF $f$ on $\left[z_{0}, \omega(F)\right]$ for some $z_{0}<\omega(F)$ can be written in the form of (53), then the corresponding CDF $F$ belongs to the $\delta$-neighborhood $Q_{2}(\delta)$ of the Weibull distribution ${ }^{8}$. The PDF of the $\mathrm{RV} \hat{\gamma}=-\gamma$ from [51] is given by (54), where $K_{1}=\frac{\theta^{\left(m+\sum_{i=1}^{N} \mu_{i}\right)} \Gamma\left[\mu+\sum_{i=1}^{N} \mu_{i}\right]}{\lambda^{m} \Gamma[\mu] \Gamma\left[\sum_{i=1}^{N} \mu_{i}\right] \prod_{i=1}^{N} \theta_{i}^{\mu_{i}-m_{i}} \lambda_{i}^{m_{i}}}$. The $E_{D}^{(2 N)}($.$) term in (54) has the following series expansion$ from [67]:

$$
\begin{gathered}
{ }_{(1)}^{(1)} E_{D}^{(N)}\left[a, b_{1}, \cdots, b_{N} ; c, c^{\prime} ; x_{1}, \cdots, x_{N}\right]= \\
\sum_{p_{1}, \cdots, p_{N}=0}^{\infty} \frac{(a)_{p_{1}+\cdots+p_{N}} \prod_{i=1}^{N}\left(b_{i}\right)_{p_{i}} \prod_{i=1}^{N} x_{i}^{p_{i}}}{(c)_{p_{1}}\left(c^{\prime}\right)_{p_{2}+\cdots+p_{N}} p_{1} ! \cdots p_{N} !} .
\end{gathered}
$$

Using the above series expansion, we rewrite (54) as

\footnotetext{
${ }^{8}$ For a real or complex valued function $g_{1}(x)$ and a strictly positive real valued function $g_{2}(x)$ both defined on some unbounded subset of $\mathbb{R}^{+}$, we say $g_{1}(x)=O\left(g_{2}(x)\right)$, iff $\exists M \in \mathbb{R}^{+}$and $x_{0} \in \mathbb{R}$ such that, $\left|g_{1}(x)\right| \leq$ $M g_{2}(x) \forall x \geq x_{0}$.
}

$$
\begin{gathered}
f_{\gamma}(z)=K_{1}(-z)^{-\mu}\left(\frac{\theta_{1}}{\theta-z \theta_{1}}\right)^{\left(\mu+\sum_{i=1}^{N} \mu_{i}\right)} \\
\sum_{p_{1}, \cdots, p_{2_{N}=0}}^{\infty} \frac{\left(\mu+\sum_{i=1}^{N} \mu_{i}\right)_{p_{1}+\cdots+p_{2 N}}}{(\mu)_{p_{1}}} \times \\
\frac{(m)_{p_{1}} \prod_{i=2}^{N}\left(\mu_{i}-m_{i}\right)_{p_{i}} \prod_{i=N+1}^{2 N}\left(m_{i}\right)_{p_{i}}}{\left(\sum_{i=1}^{N} \mu_{i}\right)_{p_{2}+\cdots+p_{2 N}}} \prod_{i=1}^{2 N} \frac{z_{i}^{p_{i}}}{p_{i} !}
\end{gathered}
$$

where $z_{1}=\frac{(\lambda-\theta)(-z) \theta_{1}}{\lambda\left(\theta-z \theta_{1}\right)}, z_{i}=\frac{\theta\left(\theta_{i}-\theta_{1}\right)}{\theta_{i}\left(\theta-z \theta_{1}\right)}$ for $i \in\{2, \cdots N\}$ and $z_{i}=\frac{\theta\left(\lambda_{i}-\theta_{1}\right)}{\lambda_{i}\left(\theta-z \theta_{1}\right)}$ for $i \in\{N+1, \cdots, 2 N\}$. We then expand the $2 N$ fold summation in (56) into three terms: the first term with all the iterating variables $p_{1}, p_{2}, \ldots, p_{2 N}$ taking the value zero, the second term with exactly one non-zero iterating variable and the third term with the rest. By expanding, (56) becomes the expression given in (60) where $\rho=\mu+\sum_{i=1}^{N} \mu_{i}$. Now, the term $\left(\frac{\theta}{\theta-z \theta_{1}}\right)^{p_{j}}$ present in Term $b$ of (60) has the following series expansion:

$$
\begin{aligned}
\left(\frac{\theta}{\theta-z \theta_{1}}\right)^{p_{j}}= & 1+\frac{p_{j} \theta_{1}}{\theta} z+\frac{\theta_{1}^{2}\left(p_{j}+p_{j}^{2}\right)}{2 \theta^{2}} z^{2}+ \\
& \frac{\left(2 p_{j}+3 p_{j}^{2}+p_{j}^{3}\right) \theta_{1}^{3}}{6 \theta^{3}} z^{3}+\mathcal{O}\left(z^{4}\right) .
\end{aligned}
$$

Similarly, the term $\left(\frac{-z \theta_{1}}{\theta-z \theta_{1}}\right)^{p_{1}}$ has the following series expansion :

$$
\begin{aligned}
\left(\frac{-z \theta_{1}}{\theta-z \theta_{1}}\right)^{p_{1}}= & \left(\frac{-z \theta_{1}}{\theta}\right)^{p_{1}}\left\{1+\frac{p_{1} \theta_{1} z}{\theta}+\frac{\theta_{1}^{2}\left(p_{1}+p_{1}^{2}\right) z^{2}}{2 \theta^{2}}\right. \\
& \left.+\frac{\theta_{1}^{3}\left(2 p_{1}+3 p_{1}^{2}+p_{1}^{3}\right) z^{3}}{6 \theta^{3}}+\mathcal{O}\left(z^{4}\right)\right\} .
\end{aligned}
$$

Thus, Term (a) will have all powers of $z \geq 1$ and Term (b) will have all powers of $z \geq 0$. Similarly, we can see that Term 3 will have all powers of $z \geq 2$. Thus, we can rewrite the pdf expression as follows :

$$
f_{Z}(z)=K_{1}(-z)^{-\mu}\left(1+K_{2}(-z)+\mathcal{O}(-z)^{2}\right)
$$

where $K_{2}$ will be a term independent of $z$. Comparing (59) with (53) and substituting $\omega(F)=0$, we can observe that the pdf of $\hat{\gamma}$ belongs to the domain of attraction of the reversed Weibull distribution with $\nu \times \delta=1$. Thus, we have $\delta=\mu^{-1}$.

Now that we have identified the $\delta$ neighbourhood for $F_{\gamma}(z)$, we make use of the following lemma from [66] to conclude the proof.

Lemma 2. Suppose that the CDF F (of i.i.d. $R V s z_{1}, \cdots, z_{K}$ ) is in the $\delta$ neighborhood $Q_{2}(\delta)$ of the GPD $W_{2, \nu}$ then there obviously exist constant $a>0$ such that $f(z)=$ $\left.\left.\frac{1}{a} W_{2, \nu}^{\prime}\left(\frac{z-\omega(F)}{a}\right)\left(1+O\left(\left(1-W_{2, \nu}(z-\omega(F))\right)^{\delta}\right)\right\} W_{2, \nu}(z)\right)^{\delta}\right)$ 


$$
\begin{gathered}
f_{\gamma}(z)=K_{1}(-z)^{-\mu}\left(\frac{\theta_{1}}{\theta-z \theta_{1}}\right)^{\left(\mu+\sum_{i=1}^{N} \mu_{i}\right)} \times_{(1)}^{(1)} E_{D}^{(2 N)}\left[\mu+\sum_{i=1}^{N} \mu_{i}, m_{2}-\mu_{2}, \cdots, \mu_{N}-m_{N}, m_{1}, \cdots, m_{N} ;\right. \\
\left.\mu, \sum_{i=1}^{N} \mu_{i} ; \frac{-z \theta_{1}(\lambda-\theta)}{\lambda\left(\theta-z \theta_{1}\right)}, \frac{\theta\left(\theta_{2}-\theta_{1}\right)}{\theta_{2}\left(\theta-z \theta_{1}\right)}, \cdots, \frac{\theta\left(\theta_{N}-\theta_{1}\right)}{\theta_{N}\left(\theta-z \theta_{1}\right)}, \frac{\theta\left(\lambda_{1}-\theta_{1}\right)}{\lambda_{1}\left(\theta-z \theta_{1}\right)}, \cdots, \frac{\theta\left(\lambda_{N}-\theta_{1}\right)}{\lambda_{N}\left(\theta-z \theta_{1}\right)}\right]
\end{gathered}
$$

for all $z$ in the left neighborhood of $\omega\left(W_{2, \nu}\right)$. Consequently we have,

$$
\begin{aligned}
\sup _{B \in \mathbb{B}} \mid \mathbb{P}\left(\left(\left(\frac{M_{K}}{a}\right) / K^{\nu}\right)\right. & \in B)-G_{\nu}(B) \mid= \\
O & \left(\left(\frac{1}{K}\right)^{\delta}+\frac{1}{K}\right),
\end{aligned}
$$

where $\mathbb{B}$ denotes the Borel $\sigma$ algebra on $\mathbb{R}$ and $M_{K}=$ $\max \left\{z_{1}, \cdots, z_{K}\right\}$.

Since the $\mathrm{CDF} F_{\gamma}(z)$ belongs to the $\delta$ neighborhood of $Q_{2}(\delta)$, by the previous lemma, the rate of convergence is $O\left(\left(\frac{1}{K}\right)^{\delta}+\frac{1}{K}\right)$ with $\delta=\mu^{-1}$.

\section{REFERENCES}

[1] D. Datla, A. M. Wyglinski, and G. J. Minden, "A spectrum surveying framework for dynamic spectrum access networks," IEEE Trans. on Veh. Tech., vol. 58, no. 8, pp. 4158-4168, Oct 2009.

[2] J. Wang, M. Ghosh, and K. Challapali, "Emerging cognitive radio applications: A survey," IEEE Commun. Mag., vol. 49, no. 3, pp. 74-81, March 2011.

[3] Y. Liang, K. Chen, G. Y. Li, and P. Mahonen, "Cognitive radio networking and communications: an overview," IEEE Trans. on Veh. Tech., vol. 60, no. 7, pp. 3386-3407, Sep. 2011.

[4] A. Goldsmith, S. A. Jafar, I. Maric, and S. Srinivasa, "Breaking spectrum gridlock with cognitive radios: an information theoretic perspective," Proc. of the IEEE, vol. 97, no. 5, pp. 894-914, May 2009.

[5] N. I. Miridakis, T. A. Tsiftsis, and G. C. Alexandropoulos, "Mimo underlay cognitive radio: Optimized power allocation, effective number of transmit antennas and harvest-transmit tradeoff," IEEE Trans. Green Commun. Netw., vol. 2, no. 4, pp. 1101-1114, 2018.

[6] Q. Zhao and B. M. Sadler, "A survey of dynamic spectrum access," IEEE Sig. Proc. Mag., vol. 24, no. 3, pp. 79-89, May 2007.
[7] M. G. Khoshkholgh, K. Navaie, and H. Yanikomeroglu, "Access strategies for spectrum sharing in fading environment: overlay, underlay, and mixed," IEEE Trans. on Mob. Comput., vol. 9, no. 12, pp. 1780-1793, Dec 2010.

[8] A. Patel, M. Z. A. Khan, S. N. Merchant, U. B. Desai, and L. Hanzo, "The achievable rate of interweave cognitive radio in the face of sensing errors," IEEE Access, vol. 5, pp. 8579-8605, 2017.

[9] A. Patel, M. Z. A. Khan, S. Merchant, U. Desai, and L. Hanzo, "How many cognitive channels should the primary user share?" IEEE Wireless Commun., no. 99, pp. 1-8, 2018.

[10] Y. Zou, B. Champagne, W.-P. Zhu, and L. Hanzo, "Relay-selection improves the security-reliability trade-off in cognitive radio systems," IEEE Trans. Commun., vol. 63, no. 1, pp. 215-228, 2015.

[11] X. Ding, Y. Zou, G. Zhang, X. Chen, X. Wang, and L. Hanzo, "The security-reliability tradeoff of multiuser scheduling aided energy harvesting cognitive radio networks," IEEE Trans. Commun., 2019.

[12] R. Zhang, "On peak versus average interference power constraints for protecting primary users in cognitive radio networks," IEEE Trans. on Wireless Commun., vol. 8, no. 4, pp. 2112-2120, April 2009.

[13] H. A. Suraweera, P. J. Smith, and M. Shafi, "Capacity limits and performance analysis of cognitive radio with imperfect channel knowledge," IEEE Trans. on Veh. Tech., vol. 59, no. 4, pp. 1811-1822, May 2010.

[14] Z. Rezki and M. Alouini, "Ergodic capacity of cognitive radio under imperfect channel-state information," IEEE Trans. Veh. Tech., vol. 61, no. 5, pp. 2108-2119, Jun 2012.

[15] X. Kang, R. Zhang, Y. Liang, and H. K. Garg, "Optimal power allocation strategies for fading cognitive radio channels with primary user outage constraint," IEEE J. Sel. Areas Commun., vol. 29, no. 2, pp. 374-383, February 2011.

[16] P. J. Smith, P. A. Dmochowski, H. A. Suraweera, and M. Shafi, "The effects of limited channel knowledge on cognitive radio system capacity," IEEE Trans. Veh. Tech., vol. 62, no. 2, pp. 927-933, Feb 2013.

[17] M. Hanif, H. Yang, and M. Alouini, "Transmit antenna selection for power adaptive underlay cognitive radio With instantaneous interference constraint," IEEE Trans. Commun., vol. 65, no. 6, pp. 2357-2367, June 2017.

[18] A. Patel, M. Z. A. Khan, S. Merchant, U. B. Desai, and L. Hanzo, "Achievable rates of underlay-based cognitive radio operating under rate limitation," IEEE Trans. Veh. Tech., vol. 65, no. 9, pp. 7149-7159, 2016.

[19] L. Sibomana and H.-J. Zepernick, "Ergodic capacity of multiuser 
scheduling in cognitive radio networks: analysis and comparison," Wireless Communications and Mobile Computing, vol. 16, no. 16, pp. 2759-2774, 2016.

[20] Y. H. Al-Badarneh, C. N. Georghiades, and M. Alouini, "Asymptotic performance analysis of generalized user selection for interferencelimited multiuser secondary networks," IEEE Trans. Cog. Commun. and Netw., pp. 1-1, 2019.

[21] C.-S. Chang, "Stability, queue length, and delay of deterministic and stochastic queueing networks," IEEE Trans. Autom. Control, vol. 39, no. 5, pp. 913-931, 1994.

[22] Y. Wang, X. Tang, and T. Wang, "A unified qos and security provisioning framework for wiretap cognitive radio networks: A statistical queueing analysis approach," IEEE Trans. Wireless Commun., vol. 18, no. 3, pp. $1548-1565,2019$

[23] Y. Zou, X. Li, and Y.-C. Liang, "Secrecy outage and diversity analysis of cognitive radio systems," IEEE J. Sel. Areas Commun., vol. 32, no. 11, pp. 2222-2236, 2014.

[24] H. Lei, C. Gao, I. S. Ansari, Y. Guo, Y. Zou, G. Pan, and K. A. Qaraqe, "Secrecy outage performance of transmit antenna selection for mimo underlay cognitive radio systems over nakagami- $m$ channels," IEEE Trans. on Veh. Tech., vol. 66, no. 3, pp. 2237-2250, 2016.

[25] M. Bouabdellah, F. El Bouanani, and H. Ben-Azza, "Secrecy outage probability in cognitive radio networks subject to rayleigh fading channels," in 2018 Int. Conf. Adv. Commun. Tech. Netw. (CommNet). IEEE, 2018, pp. 1-5.

[26] M. Yacoub, "The $\kappa-\mu$ distribution and the $\eta-\mu$ distribution," IEEE Antennas and Propagat. Mag., vol. 49, no. 1, pp. 68-81, Feb 2007.

[27] J. F. Paris, "Statistical characterization of $\kappa-\mu$ shadowed fading," IEEE Trans. Veh. Tech., vol. 63, no. 2, pp. 518-526, 2014.

[28] S. L. Cotton, "Human body shadowing in cellular device-to-device communications: channel modeling using the shadowed $\kappa-\mu$ fading model," IEEE Journal of Sel. Topics in Comm., vol. 33, no. 1, pp. 111119, Jan 2015.

[29] L. Moreno-Pozas, F. J. Lopez-Martinez, J. F. Paris, and E. MartosNaya, "The $\kappa-\mu$ shadowed fading model: unifying the $\kappa-\mu$ and $\eta-\mu$ distributions," IEEE Trans. Veh. Tech., vol. 65, no. 12, pp. 9630-9641, Dec 2016.

[30] C. Garca-Corrales, F. J. Caete, and J. F. Paris, "Capacity of $\kappa-\mu$ shadowed fading channels," International Journal of Antennas and Propagation, 2014.

[31] J. Zhang, L. Dai, W. H. Gerstacker, and Z. Wang, "Effective capacity of communication systems over $\kappa-\mu$ shadowed fading channels," Electron. Lett., vol. 51, no. 19, pp. 1540-1542, 2015.

[32] C. Chen, M. Shu, Y. Wang, and C. Zhang, "Outage probability analysis for MRC in $\kappa-\mu$ shadowed fading channels with co-channel interference," in IEEE Int. Conf. on Info. and Autom., Aug 2016, pp. 270-275.

[33] X. Li, J. Li, L. Li, J. Jin, J. Zhang, and D. Zhang, "Effective rate of MISO systems over $\kappa-\mu$ shadowed fading channels," IEEE Access, vol. 5, pp. 10605-10611, 2017.

[34] J. Zhang, X. Chen, K. P. Peppas, X. Li, and Y. Liu, "On high-order capacity statistics of spectrum aggregation systems over $\kappa-\mu$ and $\kappa-\mu$ shadowed fading channels," IEEE Trans. on Comm., vol. 65, no. 2, pp. 935-944, Feb 2017.

[35] G. Chandrasekaran and S. Kalyani, "Performance analysis of cooperative spectrum sensing over $\kappa-\mu$ shadowed fading," IEEE Wireless Commun. Lett., vol. 4, no. 5, pp. 553-556, 2015.

[36] V. A. Thomas, S. Kumar, S. Kalyani, M. El-Hajjar, K. Giridhar, and L. Hanzo, "Error vector magnitude analysis of fading SIMO channels relying on MRC reception," IEEE Trans. Commun., vol. 64, no. 4, pp. 1786-1797, 2016.

[37] D. Morales-Jimenez, J. F. Paris, and A. Lozano, "Outage probability analysis for MRC in $\eta$ - $\mu$ fading channels with co-channel interference," IEEE Commun. Lett., vol. 16, no. 5, pp. 674-677, May 2012.

[38] J. F. Paris, "Outage Probability in $\eta-\mu / \eta-\mu$ and $\kappa-\mu / \eta-\mu$ interferencelimited Scenarios," IEEE Trans. Commun., vol. 61, no. 1, pp. 335-343, January 2013.

[39] N. Y. Ermolova and O. Tirkkonen, "Outage probability analysis in generalized fading channels with co-channel interference and background noise: $\eta-\mu / \eta-\mu, \eta-\mu / \kappa-\mu$, and $\kappa-\mu / \eta-\mu$ scenarios," IEEE Trans. Wireless Commun., vol. 13, no. 1, pp. 291-297, January 2014.

[40] S. Kumar and S. Kalyani, "Coverage probability and rate for $\kappa-\mu / \eta-\mu$ fading channels in interference-limited scenarios," IEEE Trans. Wireless Commun., vol. 14, no. 11, pp. 6082-6096, Nov 2015.

[41] S. Kumar, G. Chandrasekaran, and S. Kalyani, "Analysis of outage probability and capacity for $\kappa-\mu / \eta-\mu$ faded channel," IEEE Commun. Lett., vol. 19, no. 2, pp. 211-214, Feb 2015.
[42] J. Zhang, X. Li, I. S. Ansari, Y. Liu, and K. A. Qaraqe, "Performance analysis of dual-hop DF satellite relaying over $\kappa-\mu$ shadowed fading channels," in Proc. IEEE Wireless Commun. \& Netw. Conf. IEEE, 2017, pp. 1-6.

[43] S. Parthasarathy and R. K. Ganti, "Coverage analysis in downlink poisson cellular network with $\kappa-\mu$ shadowed fading," IEEE Wireless Commun. Lett., vol. 6, no. 1, pp. 10-13, Feb 2017.

[44] S. Parthasarathy, S. Kumar, R. K. Ganti, S. Kalyani, and K. Giridhar, "Error vector magnitude analysis in generalized fading with co-channel interference," IEEE Trans. Commun., vol. 66, no. 1, pp. 345-354, Jan 2018.

[45] M. Srinivasan and S. Kalyani, "Secrecy capacity of $\kappa-\mu$ shadowed fading channels," IEEE Commun. Lett., vol. 22, no. 8, pp. 1728-1731, Aug 2018.

[46] S. Kumar and S. Kalyani, "Outage probability and rate for $\kappa-\mu$ shadowed fading in interference limited scenario," IEEE Trans. Wireless Commun., vol. 16, no. 12, pp. 8289-8304, 2017.

[47] B. Aghazadeh and M. Torabi, "Performance evaluation of multi-user diversity in a SIMO spectrum sharing system with reduced CSI load," Digital Signal Processing, vol. 72, pp. 160-170, 2018.

[48] F. A. Khan, K. Tourki, M.-S. Alouini, and K. A. Qaraqe, "Performance analysis of an opportunistic multi-user cognitive network with multiple primary users," Wireless Commun. Mob. Comput., vol. 15, no. 16, pp. 2004-2019, 2015.

[49] D. Xu and Q. Li, "Resource allocation for cognitive radio with primary user secrecy outage constraint," IEEE Sys. J., vol. 12, no. 1, pp. 893-904, 2016.

[50] Y. Wang, P. Ren, Q. Du, and L. Sun, "Optimal power allocation for underlay-based cognitive radio networks with primary user's statistical delay qos provisioning," IEEE Trans. Wireless Commun., vol. 14, no. 12, pp. 6896-6910, Dec 2015.

[51] A. Subhash, M. Srinivasan, and S. Kalyani, "Asymptotic maximum order statistic for sir in $\kappa-\mu$ shadowed fading," IEEE Trans. Commun., 2019.

[52] T. W. Ban, W. Choi, B. C. Jung, and D. K. Sung, "Multi-user diversity in a spectrum sharing system," IEEE Trans. Wireless Commun., vol. 8, no. 1 , pp. 102-106, 2009.

[53] C.-J. Chen and L.-C. Wang, "A unified capacity analysis for wireless systems with joint multiuser scheduling and antenna diversity in nakagami fading channels," IEEE Trans. Commun., vol. 54, no. 3, pp. 469-478, 2006.

[54] G. Song and Y. Li, "Asymptotic throughput analysis for channel-aware scheduling," IEEE Trans. Commun., vol. 54, no. 10, pp. 1827-1834, 2006.

[55] S. Kumar and S. Kalyani, "Errata to the paper Outage Probability and Rate for $\kappa-\mu$ Shadowed Fading in Interference Limited Scenario," IEEE Trans. Wireless Commun., 2019.

[56] "Code for evaluating fnd," http://faculty.smu.edu/rbutler/, Accessed: 2018 [Online].

[57] Dapeng Wu and R. Negi, "Effective capacity: a wireless link model for support of quality of service," IEEE Trans. Wireless Commun., vol. 2, no. 4, pp. 630-643, July 2003.

[58] L. H. Ozarow and A. D. Wyner, "Wire-tap channel ii," AT\&T Bell Laboratories technical journal, vol. 63, no. 10, pp. 2135-2157, 1984.

[59] T. X. Quach, H. Tran, E. Uhlemann, G. Kaddoum, and Q. A. Tran, "Power allocation policy and performance analysis of secure and reliable communication in cognitive radio networks," Wireless Networks, vol. 25, no. 4, pp. 1477-1489, 2019.

[60] D.-K. Jeong and D. Kim, "Power allocation and outage analysis for cognitive radio with jamming signal under primary secrecy outage constraint," in 2018 28th International Telecommunication Networks and Applications Conference (ITNAC). IEEE, 2018, pp. 1-6.

[61] J. Ji and W. Chen, "Capacity analysis of multicast transmission schemes in a spectrum-sharing scenario," IET Communications, vol. 6, no. 17, pp. 2974-2979, 2012.

[62] ITU-R, "Requirements related to technical performance for IMTAdvanced radio interface (s)," International Telecommunications Union, 2008.

[63] S. Sesia, M. Baker, and I. Toufik, LTE-the UMTS long term evolution: from theory to practice. John Wiley \& Sons, 2011.

[64] E. J. Gumbel, Statistics of extremes. Courier Corporation, 2012.

[65] L. De Haan and A. Ferreira, Extreme value theory: an introduction. Springer Science \& Business Media, 2007.

[66] M. Falk, J. Hüsler, and R.-D. Reiss, Laws of small numbers: extremes and rare events. Springer Science \& Business Media, 2010.

[67] H. Exton, "Multiple hypergeometric functions and applications," 1976. 


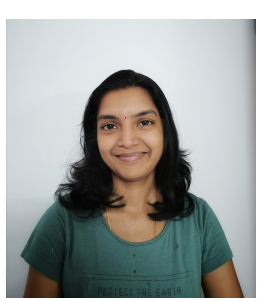

Athira Subhash received the B.Tech degree in electronics and communication engineering and the M.Tech degree in Computational Engineering and Networking from Amrita School of Engineering, Amrita Vishwa Vidyapeetham University. She is currently working toward the Ph.D. degree in electrical engineering with the Indian Institute of Technology Madras, Chennai, India. Her research interests include extreme value theory, performance analysis and resource allocation of wireless communication systems.

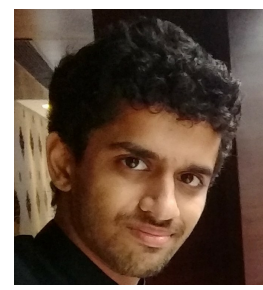

Muralikrishnan Srinivasan received the B.E. degree in electronics and communication engineering from the College of Engineering - Guindy, Anna University, Chennai in 2012. He is currently working towards the Ph.D. degree in electrical engineering at the Indian Institute of Technology, Madras, Chennai, India. His research interests include generalized fading models, hypergeometric functions, performance analysis of wireless systems, resource allocation and deep learning.

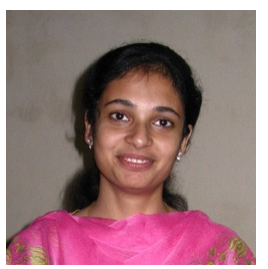

Sheetal Kalyani received the B.E. degree in electronics and communication engineering from the Sardar Patel University, Gujarat India in 2002 and the Ph.D. degree in electrical engineering from the Indian Institute of Technology, Madras, India in 2008. She was a Senior Research Engineer in Centre of Excellence in Wireless Technology, Chennai India from 2008 to 2012. She is is currently an Associate Professor in the Dept. of Electrical Engineering in IIT Madras. Her current research interests include extreme value theory, generalized fading models, hypergeometric functions, performance analysis of wireless systems/networks, compressed sensing, machine learning and deep learning for wireless applications.

Lajos Hanzo ("http://www-mobile.ecs.soton.ac. uk,https://en.wikipedia.org/wiki/Lajos_Hanzo"), FREng, FIEEE, FIET, Fellow of EURASIP, DSc holds an honorary doctorate by the Technical University of Budapest (2009) and by the University of Edinburgh (2015). He is a Foreign Member of the Hungarian Academy of Sciences and a former Editor-in-Chief of the IEEE Press. He has served as Governor of both IEEE ComSoc and of VTS. He has published $1900+$ contributions at IEEE Xplore, 19 Wiley-IEEE Press books and has helped the fast-track career of $119 \mathrm{PhD}$ students. Over 40 of them are Professors at various stages of their careers in academia and many of them are leading scientists in the wireless industry. 